\begin{abstract}
Title of Document:

DIRECT OBSERVATION OF AMYLOID

NUCLEATION UNDER NANOMECHANICAL

STRETCHING

Nitinun Varongchayakul,

Master of Science 2013

Directed By:

Assistant Professor, Joonil Seog, Materials Science and Engineering
\end{abstract}

Self-assembly of amyloid nanofiber is associated with functional and pathological processes such as in neurodegenerative diseases. Despite intensive studies, stochastic nature of the process has made it difficult to elucidate molecular mechanisms for the key amyloid nucleation. Here, we investigated the amyloid nucleation of silk-elastinlike peptide (SELP) using time-lapse lateral force microscopy (LFM). By repeated scanning a single line on a SELP-coated mica surface, we observed sudden stepwise height increases, corresponds to nucleation of an amyloid fiber. The lateral force profiles followed either a worm-like chain model or an exponential function, suggesting that the atomic force microscopy (AFM) tip stretches a single or multiple SELP molecules along the scanning direction, serves as the template for further selfassembly perpendicular to the scan direction. Such mechanically induced nucleation of amyloid fibrils allows positional and directional control of amyloid assembly in vitro, which we demonstrate by generating single nanofibers at predetermined nucleation sites. 


\title{
DIRECT OBSERVATION OF AMYLOID NUCLEATION UNDER NANOMECHANICAL STRETCHING
}

\author{
By \\ Nitinun Varongchayakul \\ Thesis submitted to the Faculty of the Graduate School of the \\ University of Maryland, College Park, in partial fulfillment \\ of the requirements for the degree of \\ Master of Science \\ 2013
}

Advisory Committee:

Assistant Professor Joonil Seog, Chair

Professor Manfred Wuttig

Professor Mohamed Al-Sheikhly

Associate Professor Santiago Solares 
(C) Copyright by

Nitinun Varongchayakul

2013 


\section{Dedication}

I dedicate my thesis work for the greatest love from another part of the world, from my family. A special gratitude goes towards my parents, Chairoj and Wimol Varongchayakul, and my lovely sister, Chanokporn, who remind me that after this hard work, there are a lot of good foods waiting for me at home.

I also want to dedicate this work to Eddy Salgado, my best friend that helped me struggle through hard time and listened to my pointless complaints. Portion of this work goes to my Thai "family" in Maryland, Joe, Pi Khang, Pi Sith, and Pi Mim my best roommate ever.

Finally, this work is dedicated to my first boss, tough mentor, and beloved advisor, Professor Joonil Seog. Thank you for his high expectation that I didn't think I could make it, and yet I haven't made it. 


\section{Acknowledgements}

I would like to show my gratitude towards my committee members who spent their expertise and precious time. A special thank is for Professor Joonil Seog, my committee chairman for his numerous hours of meeting, sharpening this work and encouraging me to finish this work. I also would like to thank Professor Manfred Wuttig, Prof Mohamad Al-Sheikhly, and Prof Santiago Solares, for serving as my thesis committee. Special thanks for Professor Robert Briber for revising my presentation.

Furthermore, I would like to acknowledge my collaborators, Professor Wonmuk Hwang, Professor Santiago Solares, and Professor Sameer Shah. I also would like to acknowledge undergraduate students who worked with me on this project; Sara Johnson, Trina Quabili, Stephanie Okorafor, and Louis Born as well as Post-doc Young Kwon Ko and graduate student Jonathan Chang. I give thanks to Amy Lee, Brian Stock, Elliot Bartis, and Eddy Salgado for proofreading all of my manuscripts.

Finally, I would like to acknowledge Department of Materials Science and Engineering for the opportunity of studying here. Also I appreciate School of Engineering for recognition of this work as 1st place Dean's Graduate Research Award 2013. 


\section{Table of Contents}

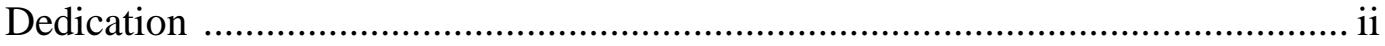

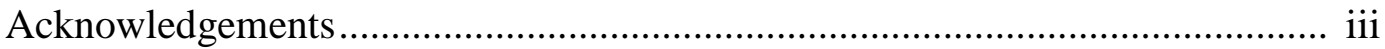

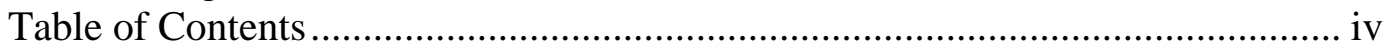

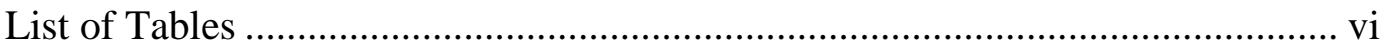

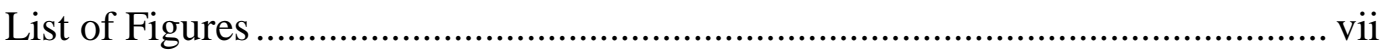

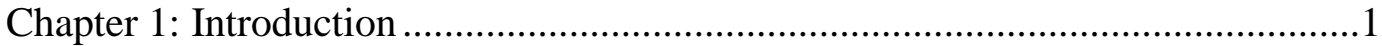

1.1 Amyloid nanofiber ...................................................................................... 1

1.2 Mechanical Effect of Amyloid Formation ..................................................2

1.3 Friction on Biomolecular Film ............................................................

1.4 Silk-Elastin-Like Peptide (SELP) ........................................................

Chapter 2: Geometrical Evolution of Amyloid Nucleation ......................................6

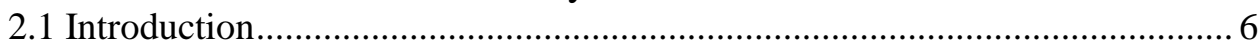

2.2 Experimental Methods..........................................................................

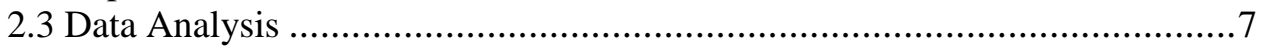

2.4 High Resolution Image Reveals Blob-Like Nanoribbon ................................ 8

2.5 Steipwise Increase in Height and Width Reflected Smallest Self-Assemble

Unit of Amyloid ...................................................................................... 10

Chapter 3: Single-Molecule Force Spectroscopy of SELP ....................................14

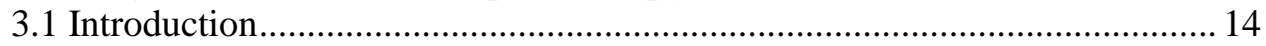

3.2 Experimental Methods........................................................................ 14

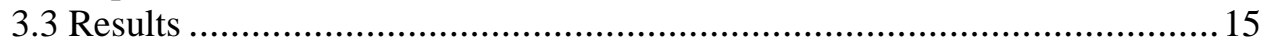

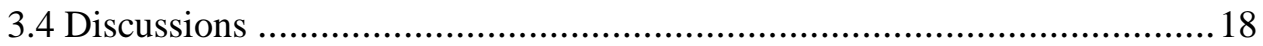

Chapter 4: Lateral Force Microscopy of SELP .................................................19

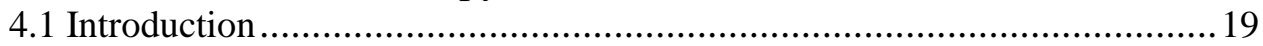

4.2 Experimental Methods...................................................................... 19

4.3 Data Analysis ............................................................................... 20

4.4 Increase in Lateral Force Results in Amyloid Nucleation .............................. 20

4.5 Increase in Lateral Force Originates From Stretching Laterally..................... 22

4.6 Stretching Force Dependency on Nucleation Formation ................................. 26

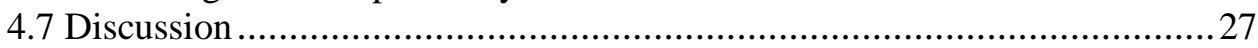

4.8 Speculation on mechanisms of nucleation formation under nanomechanical

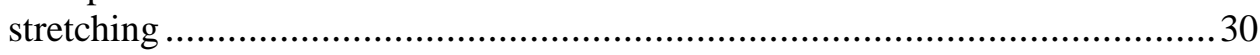

Chapter 5: Exploration of the Activation Energy of Mechanically induced

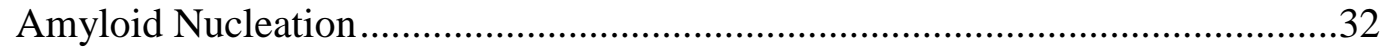

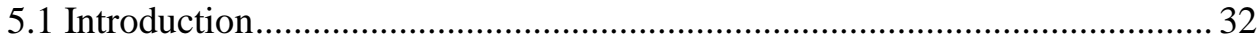

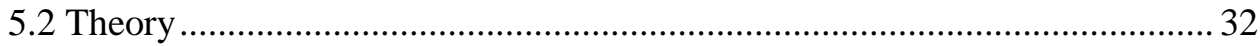

5.3 Experimental Methods............................................................................ 34

5.4 Time-Lapse Experiment: Data interpretation .................................................. 34

5.5 Normal Force Dependency on Amyloid Nucleation............................................ 35

5.6 Normal Force, Frictional Force, and Amyloid Nucleation Rate …………...... 36

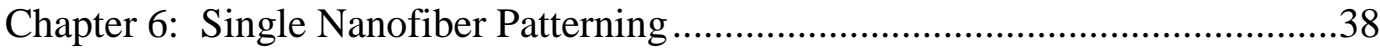

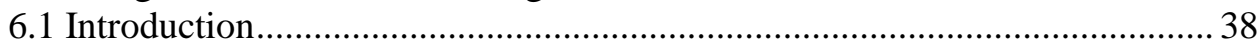

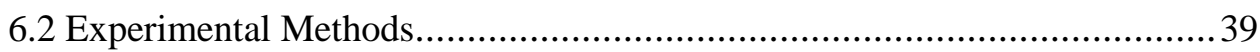

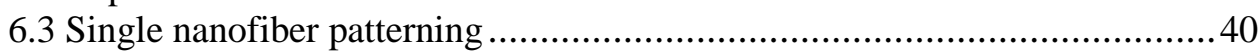

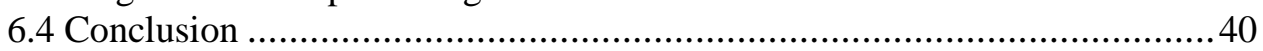


Bibliography 


\section{List of Tables}

Table 3-1 Fitting parameters in force-extension curve obtained from SELP coated

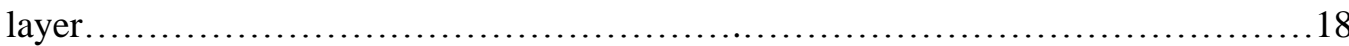

Table 5-1 Probability of nucleation at different normal force levels....................36 


\section{List of Figures}

Figure 2-1. High resolution image of amyloid nanofiber ................................... 9

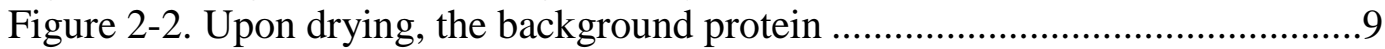

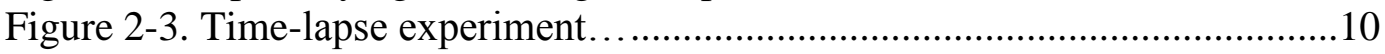

Figure 2-4. Height and width evolution of nanofiber......................................11

Figure 2-5. speculated molecular conformation of amyloid nanofiber.................13

Figure3-1. Representative normal force spectroscopy curve..............................16

Figure3-2. Representative normal force spectroscopy curves obtained at

different $\mathrm{NaCl}$ concentration........................................................................ 17

Figure 4-1. (a) A height image showing a single nucleation site formation

during a time-lapse LFM measurement......................................................21

Figure 4-2. Three types of frictional force profiles which resulted in nucleation uring time-lapse LFM measurements ...........................................................23

Figure 4-3. (a) histogram of maximum lateral force, (b) histogram of stretching

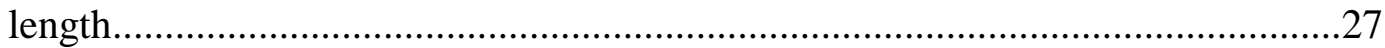

Figure 4-4. Plot of maximum stretching force vs. stretching distance ................29

Figure 4-5. Speculated molecular mechanism of nanomechanically induced amyloid nucleation............................................................

Figure 5-1. Number of scan until the nucleation site is observed is proportional to

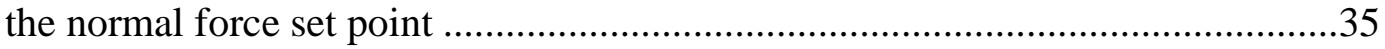

Figure 5-2. Relationship between normal force and frictional force ....................36

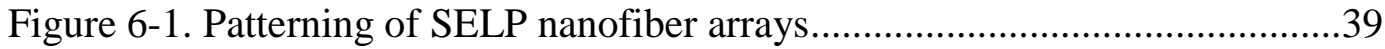

Figure 6-2. Single nanofiber pattern ..............................................................40 


\section{Chapter 1: Introduction}

\subsection{Amyloid nanofiber}

Proteins are long chain polypeptides, and usually adopt a well-defined 3D structure. They serve many important roles in bioorganisms including as enzymes, components of muscle, and are involved in many molecular processes. However, under certain conditions, proteins can undergo structural rearrangement and self-assemble into beta-sheet enriched 1D structures, with a diameter ranging from 5-10nm, which we term amyloid fibers[1]. Selfassembly of peptide molecules into fibrillar structures is an intrinsic property of some peptides in nature $[2,3]$.

Amyloid is known to be involved in pathogenic diseases as Alzheimer's, Parkinson's disease but also shows functional purposes in bacteria, fungi, and mammalian cell [4], due to its superior mechanical properties among biomaterials [5]. Furthermore, this nanofiber has potential to act as a template for nanoscale fabrication and core for nanoscale devices[6]. Therefore, it is important to understand the self-assembly process of amyloid.

Self-assembly of relatively long polypeptides is a complex process due to multiple conformational states explored by the molecule. Various conformational states are represented by highly dynamic and transient states in a rugged energy landscape [7, 8]. However, under appropriate experimental conditions, the very nature of the dynamic equilibrium between different conformational states can lead into nucleating species which subsequently form amyloids [9]. Self-assembly of amyloid is critically dependent on nucleation processes. The nucleation step is known to have a high activation energy barrier, which results in a distinct lag phase during assembly kinetics[10]. Various environmental 
factors such as $\mathrm{pH}$, ionic strength and the presence of seeds affect the lag phase of amyloid assembly[11, 12]. In addition to studies in bulk solution, amyloid formation on surfaces has been actively investigated since it may more closely mimic physiological environments such as cell membrane[13]. Surface accelerated assembly kinetics as well as directed growth of amyloid on surfaces has been reported using various peptides[14-19].

\subsection{Mechanical Effect of Amyloid Formation}

Beside $\mathrm{pH}$, ionic strength and presence of seed, mechanical force can also induce nucleation of amyloid. In macroscopic scale, sonication showed that mature fiber could be fragmented, forming broken nanofibers which serve as new seeds for further growth.[20] In other systems, sonication that provides random mechanical forces resulted in acceleration of amyloid formation by reducing a lag phase or by forming aggregated structures.[21, 22] Likewise, shear flow has been shown to induce aggregate formation which resulted in nucleation and thus amyloid. [23-25]. The flow along the axis of protein is speculated as one of the major mechanisms of ordering in the spinning apparatus of the amyloid-like fibrils from silkworms [26] to spiders [27]. Fibril fragmentation was found to be the dominant secondary process that increases the rate of fibril formation.[28] In micro/nanoscopic scale, mechanical forces applied through tapping of an AFM tip can break amyloid nanofibers on a mica surface, creating fragments which can serve as nuclei for new amyloid growth. [29]

\subsection{Friction on Biomolecular Film}

Friction on the molecular scale is one of the most complicated yet challenging in the field of material science. The study in this area is called nanotribology. Friction and shear (which is friction in fluid) are involved in many biomolecular mechanisms. For example, 
shear flow activated von Willebrand factor to partially unfold and triggers wound-healing process. [30] In addition, friction between articular cartilages results in osteoarthritis [31].

General form of friction is explained to be linearly proportional to the applied normal force known as Amontons-Coulomb law, which inevitably fails to explain microscopic friction when only a few atoms/molecules are considered. Deformation of the surface in contact as function of a normal force leads to non-linear behavior at low force regime known as Hurtzian model of contact mechanics. Further improved contact mechanics model takes into account the nonspecific adhesive interaction between two substrates known as JohnsonKendall-Roberts (JKR) and Derjaguin-Muller-Toporov (DMT) theories.

When soft matter is considered, adhesive contact mechanic is applied, however, the energy dissipation during the sliding process doesn't stem only from the elastic deformation between two surfaces (as portrayed by elastic moduli in JKR or DMT theories) but instead the energy dissipation caused by stretching or confining the polymer chain. [32] Earlier work in 19's focused on trying to explain the friction of rubbers on smooth surface. Modern work extended the model to other soft matter such as surfactant [33], alkanothiol monolayer [34], and protein films [35] [36]. In the theory, two mechanisms are incorporated. Interfacial adhesion is the mechanism that the friction is generated by chemical bond forming-breaking between two interfaces, directly related to the adhesion force between two surfaces [34], while internal cohesion refers to energy dissipation is expense for the polymer chain deforms elastically [32] [37].

Lateral force microscopy (LFM) is a powerful tool to study nanotribology. This method allows us to study the static and dynamic friction in molecular level. The tip is dragged on the surface with constant normal force and velocity, the local adhesion from atom (molecule) and the tip causes the tip to bend in lateral direction. Originally, the LFM is used 
to study the friction between two hard materials in contact, lead to the name frictional force microscopy (FFM), later on it is expands to study different behaviors of the substrate in molecular contacts, not limit to only the frictional force, including the molecular orientation and the potential field on the surface, chemical force microscopy is using the tip with modified chemistry of surface to recognize difference chemical of substrate substratum. Therefore, the name of method is generalized to LFM - the study of the lateral force.

\subsection{Silk-Elastin-Like Peptide (SELP)}

A model amyloid peptide that has recently drawn much attention is silk-elastin-like peptide polymer (SELP) [38, 39]. It consists of alternating domains of 8 silk-like units (GAGAGS), 15 elastin-like units (GVGVP), and 1 elastin unit modified by the substitution of valine for lysine (GKGVP) [38]. The elastin unit provides its thermoresponsive property and the silk unit controls the stiffness of the hydrogel which SELP forms near body temperature. We have recently discovered that SELP self-assembles into amyloid on mica surface at an ultrafast rate with virtually no lag phase [40]. We also observed that the nucleation of SELP amyloid can be accelerated by nanomechanical forces exerted during tapping mode imaging by atomic force microscopy (AFM)[41, 42]. Interestingly, the mechanically induced amyloid tends to grow perpendicular to the AFM scanning direction, implying that the growth direction is controlled by the nucleated entities created by the tapping force. This novel aspect was used to simultaneously control the location of nucleation and the growth direction to create a SELP nanofiber patterned surface. However, the molecular mechanisms underlying mechanically-induced nucleation of SELP amyloid remain to be found.

Acquiring a mechanistic understanding of the complex nucleation processes which involve multiple intermediates require direct and continuous observation. Observation of nucleation on a surface using in situ AFM therefore offers the opportunity to investigate 
nanomechanically induced amyloid nucleation directly in aqueous environments. AFM has been utilized to characterize the size, shape, kinetics and intermolecular forces of aggregating peptides on a surface in situ.[13, 43-47] In this work, we applied time-lapse lateral force microscopy (LFM) to study the nucleation mechanisms of SELP on mica surface in contact mode AFM. For higher temporal resolution, the slow scan axis was disabled and a single line was continuously scanned to observe nucleation in real time. As the tip of the AFM scans the SELP adsorbed surface, lateral force increases occasionally, indicating that strands of SELP are picked up by the tip and stretched until the bond ruptures. Stretching of SELP is followed by nucleation of an amyloid fiber growing perpendicular to the stretching direction, where we found a strong correlation between the probability of nucleation and the lateral force profiles. It thus appears that, mechanical stretching of a SELP provides a template on which the amyloid cross-beta structure can grow. Supporting this idea, the measured dimensions of the fiber agreed well with those of a molecular model of SELP amyloid. Utilizing the mechanical stimulus, we were able to induce nucleation of single fibers at prescribed location and growth direction. In addition to practical potential for positional and directional control in nanopatterning amyloid fibers, the present results may help understand how mechanical forces alter the energy landscape and guide peptides to undergo conformational transitions from disordered and loosely bound aggregates to the paracrystalline amyloid form. 


\section{Chapter 2: Geometrical Evolution of Amyloid Nucleation}

\subsection{Introduction}

Conventional AFM scan on the area takes 2-10 minutes, this time scale is greater than the protein folding which is in sub-second scale. Therefore, we overcame this time resolution limit by study only the height evolution in only one line scan, result in sub-second time scale.

High resolution image of SELP fiber obtained using Si tip (2nm in diameter) gives us the precise geometrical information about SELP supersecondary structure assembly pattern. The height variation along the fiber shows the characteristic height in stepwise manner, consistent with the height evolution of the nucleation site obtained by time-lapse line scan experiment.

\subsection{Experimental Methods}

Silk-elastinlike protein polymer (SELP) is composed of specific repeating amino acids from Bombyx mori (silkworm) silk (Gly-Ala-Gly-Ala-Gly-Ser) and mammalian elastin (Gly-Val-Gly-Val-Pro). Silk-elastin-like peptide polymer (SELP) was produced using genetic modifications described by Dandu et al [38]. One SELP unit consisted of 8 Bombyx mori silk-like (GAGAGS), 15 elastin-like(GVGVP), and one lysine-modified elastin-like (GKGVP) blocks. One polymer chain consisted of a head region, six SELP units, and a tail region. The estimated total length of the fully extended chain is $295 \mathrm{~nm}$ (818 residues). Sample preparation began with $40 \mu \mathrm{l}$ of $5 \mu \mathrm{g} / \mathrm{ml}$ SELP $815 \mathrm{~K}$ in distilled water dropped onto a freshly cleaved mica disk (Ted Pella, Inc., CA). After 30 minutes of incubation at room temperature, the sample was washed by $40 \mu \mathrm{l}$ of distilled water three times. The sample was kept covered 
with water for at least $3 \mathrm{hrs}$ to ensure that the adsorbed protein layer was stable before running a time-lapse lateral force microscopy (LFM) experiment.

The experiments were carried out on a Molecular Force Probe-3D instrument (MFP3D, Asylum Research, CA). A rectangular SiN probe (MLCT Bruker, CA) was used. The spring constant was calculated using the MFP-3D software (Igor Pro Version 6.22A, MFP3D) using the thermal tuning method, which gave a value of $30 \mathrm{pN} / \mathrm{nm}$. Time-lapse experiments were carried out in "slow-scan disabled" mode. The height and friction images were obtained as a stack of topology profiles in a single line trajectory as a function of time. The time-lapse experiments were performed for $128 \mathrm{sec}$ or 512 total scan lines per each experiment. The scan frequency was $2 \mathrm{~Hz}$, thus the spatial resolution was 0.25 seconds per line. After completion of each time-lapse frame, an area scan was obtained in the same location to verify that the nucleation site leads indeed to nanofiber elongation from that location, and that it was perpendicular to the scan direction.

High Resolution Imaging was carried out by Molecular Force Probe-3D instrument (MFP-3D, Asylum Research, CA) in water. The Si probe on triangular SiN soft cantilever (MSNL Bruker, CA) was used with the tip diameter smaller than $2 \mathrm{~nm}$ (industrial specification). The spring constant was calculated using MFP-3D software (Igor Pro Version $6.22 \mathrm{~A}, \mathrm{MFP} 3 \mathrm{D})$ using the thermal tuning method, the value is around $17 \mathrm{pN} / \mathrm{nm}$. The imaging was done in non-contact (tapping) mode in aqueous environment. High resolution images were obtained in both area and time-lapse mode.

\section{$\underline{2.3 \text { Data Analysis }}$}

In order to obtain the height of the nuclei that formed during the time-lapse experiment, the line was manually drawn on the nanofibers and extracted the average height value relative to the height of the background. The width is obtained by drawing the cross 
section perpendicular to the fiber axis and average the height profile for $5 \mathrm{~nm}$ thickness. The height profile of nanofiber was fitted with Gaussian distribution (curve fitting panel, Igor Pro Version 6.22A, MFP3D) to obtain the width-at-half-max and recorded as width of the nanofiber. Tip broadening effect was also considered in reporting the width, so the tip deconvolution was implemented using tip radius $2 \mathrm{~nm}$ (industrial specification).

$$
w=2 \cdot\left(2 R_{t} h\right)^{\frac{1}{2}}
$$

Where $\mathrm{w}$ is appearance width measured from AFM experiment. $\mathrm{R}_{\mathrm{t}}$ is the tip radius and $h$ is the actual width of the nanofiber.

\subsection{High Resolution Image Reveals Blob-Like Nanoribbon.}

High resolution images of SELP fiber deposited on mica substrate are obtained using Si tip (tip diameter 2nm, measured by Scanning Electron Microscope, SEM) instead of SiN tip to lessen the tip broadening effects. The image reveals the ribbon structure with a width $8.7 \pm 2.7 \mathrm{~nm}$ and $25 \pm 5 \mathrm{~nm}$ and height $4.5 \pm 1.3 \mathrm{~nm}$. The cross section of the smaller width fiber seems to follow Gaussian distribution while cross section of the larger width fiber is square, with a little bump in the center [48] of the fiber. This implied that the fiber that has smaller width have one unit while the wider fibers originated from two fibers running parallel to each other. When the line is drawn along the fiber axis, the height profile has large fluctuation along the fiber axis, the histogram of the height shows the different height population with the quantization of $1.7 \mathrm{~nm}$, causing the fiber looks like "bead-string structure". However, there is no characteristic spacing between those "beads". This bead-like might originate from the stacking of beta sheet along the fiber, while some region has lower stacking layers, some has more. 

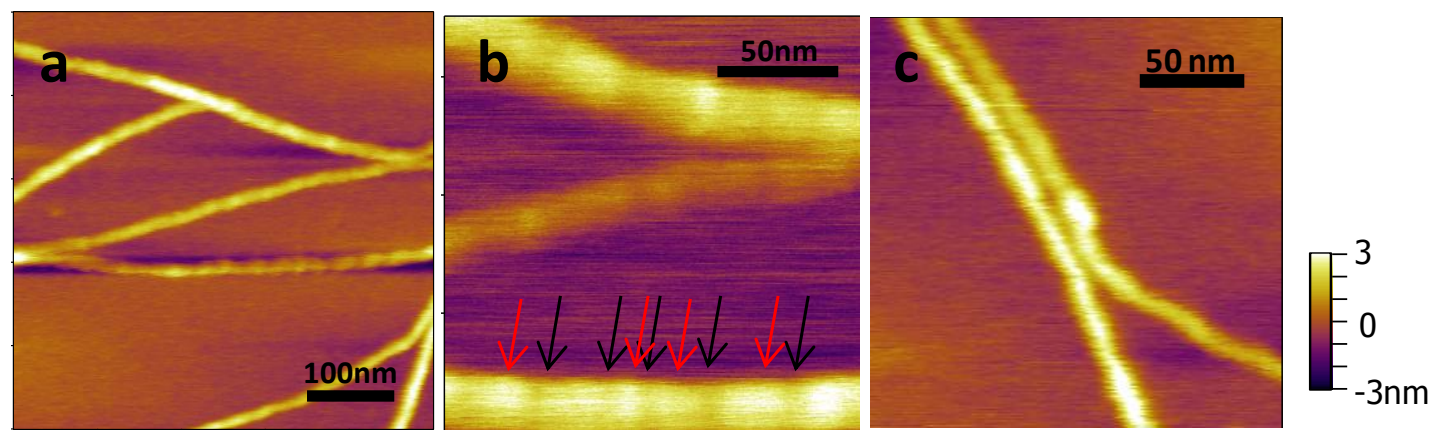

Figure 2-1. High resolution image of amyloid nanofiber. (a) the nanofiber natureally deposited on the surface and formed network-like structure. (b) Higher magnification image exhibited the blob like structure showing higher area (indicated by red arrows) and lower area (indicated by black arrow). The width of these fibers is around $25 \mathrm{~nm}$. (c) The same protein fiber from different area showing that the width of nanofiber is much smaller $(8 \mathrm{~nm})$ while the height and blob-like feature remained.

Background appears to be flat, however, force spectroscopy on the background reveals that the background consists of enriched SELP layer that even some SELP fold and form fiber, this layer didn't shows any depletion region.

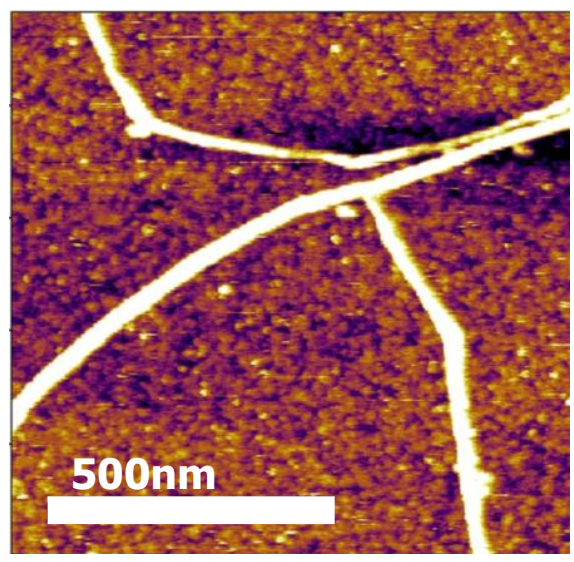

Figure 2-2. Upon drying, the background protein was revealed to consist of multiple spherelike particle of protein deposited on the surface while some underwent structural rearrangement into nanofibers (Image is taken from Jonathan Chang's Master's thesis with permission). 


\section{$\underline{\text { Amyloid }}$}

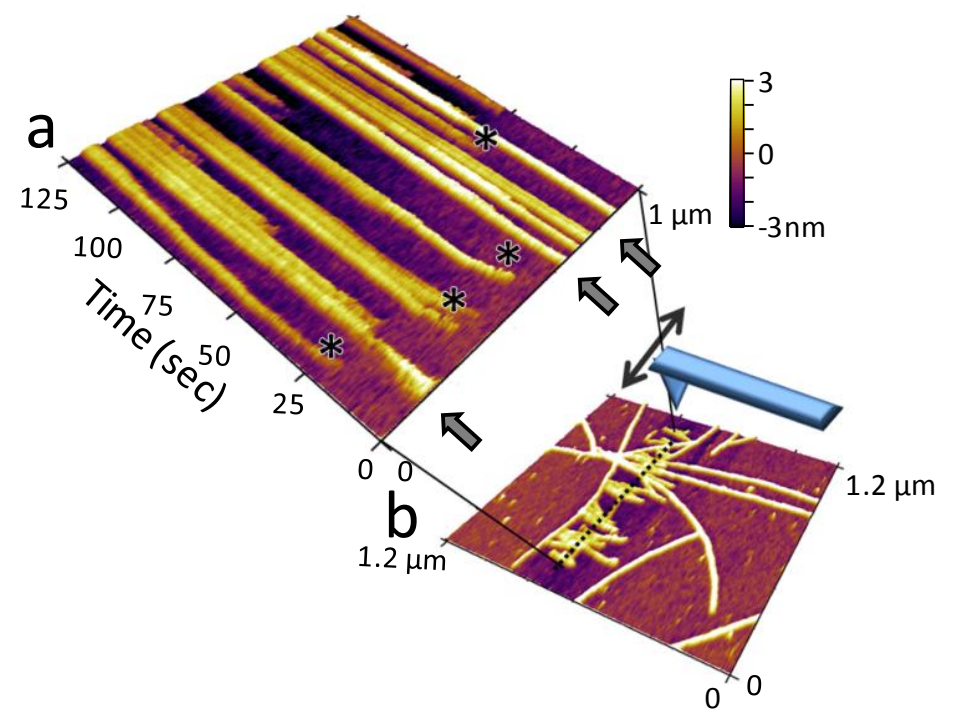

Figure 2-3. Time-lapse experiment. (a) The time-lapse of height profile. A SELP modified AFM tip was scanned on a SELP adsorbed mica surface along the same scan line repeatedly as a function of time. At $\mathrm{t}=0$, the height profile shows the heightened areas (arrows) due to the presence of pre-formed amyloid during the incubation stage. As time increased, multiple heightened sites which are indicated by asterisks were newly formed. The scan rate was 10 $\mu \mathrm{m} / \mathrm{sec}$. (b) Zoomed-out AFM image after the time-lapse LFM experiment. The dotted line indicates where the single line was repeatedly scanned. Multiple nanofibers were originated from the single scanned line, confirming that the newly formed heightened sites were indeed the nuclei for new amyloid nanofibers. (Inset) front view of tip during LFM experiment. Torsion of the cantilever occurs in response to lateral forces while normal bending of the cantilever occurs in response to normal forces. The arrow indicates the scan direction.

Figure 3 illustrates the time-lapse atomic force microscopy. At the beginning of the scan, there were heightened features as indicated by arrows due to the presence of amyloids that were pre-formed during the incubation stage (Figure 3a). Later on, multiple heightened sites appeared at different time points as indicated by asterisks. A zoomed out image of the scanned line (the dotted line in Figure $3 b$ ) indicated that nanofibers were grew from these 
locations, confirming that increase in height during the line scan correspond to nucleation events (Figure 3b).
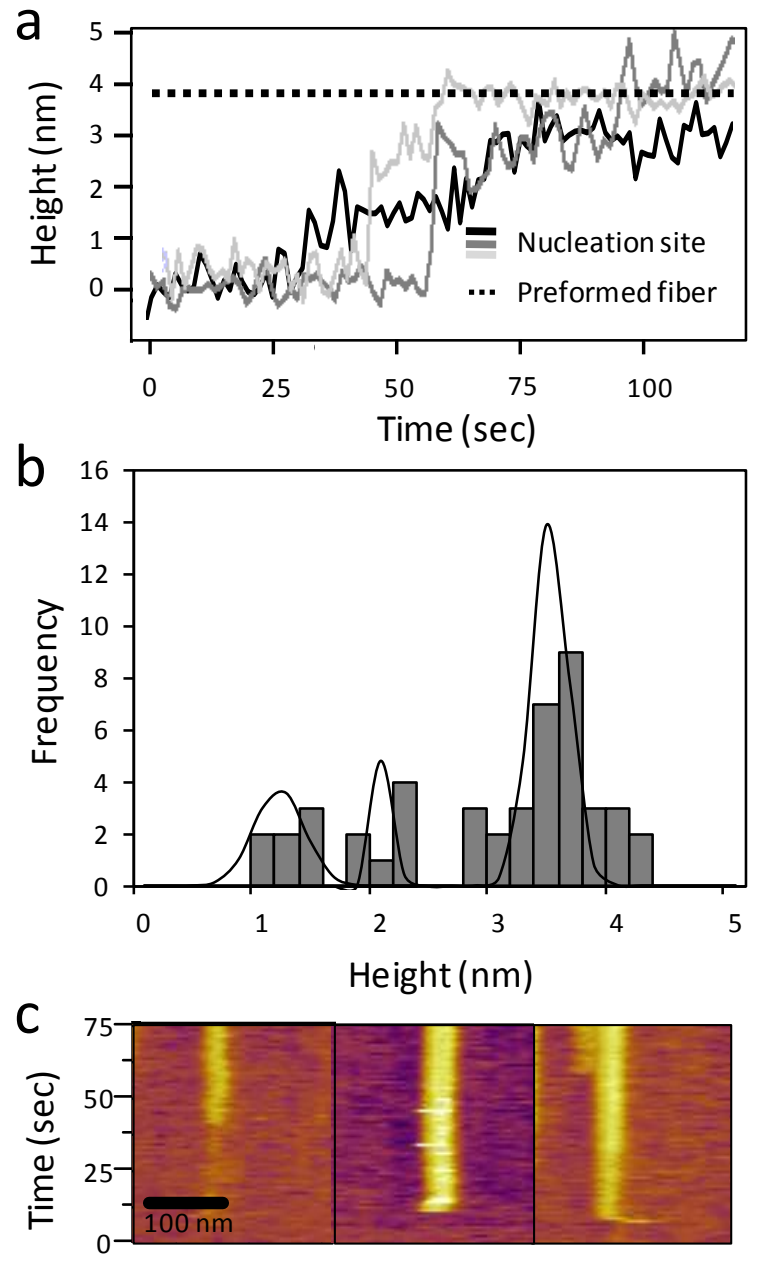

Figure 2-4. (a) Height evolution of nuclei as a function of time. A sudden stepwise increase in height occurred during time-lapse LFM. The multiple steps show a hierarchical assembly process of nucleation formation. (b) Histogram of step sizes that occurred during nucleation. The step size increase was quantized and the smallest step size was $\sim 1.2 \mathrm{~nm}$, which matches very well with the height of a single beta hairpin structure. (c) The width of mechanically induced nanofiber showed two population which due to double fibers formed parallel to each other. 
Figure 4a shows the height evolution profile of newly formed nuclei as a function of time. The height increase occurred in a stepwise manner during the scan, showing a hierarchical assembly process of amyloid nucleation. The smallest step size was $\sim 1.2 \pm 0.2 \mathrm{~nm}$ $(n=7)$ which is consistent with the dimension of one layer thickness of beta sheet structures (Figure 2b).[19] Some profiles showed a single large step increase which was quantized at $2.1 \pm 0.1 \mathrm{~nm}(\mathrm{n}=7)$ and $3.5 \pm 0.2 \mathrm{~nm}(\mathrm{n}=32)$, indicating that multiple layers of beta sheet were formed instantly. The fully grown height of the nucleus coincides with the height of preformed SELP which is $3.7 \pm 0.2 \mathrm{~nm}(\mathrm{n}=7)$. This strongly indicates that SELP amyloid is composed of multiple layers of beta sheet analogous to the structure of other amyloid nanofibers. Rarely, continuous increase of height occurred without any well-defined step size, implying that aggregation of SELP also occurred. The increase in nucleus width also showed quantized behaviors as a function of time (Figure 2c). Initially, the width of the nucleation site measured using the SELP modified tip was $19 \pm 2.4 \mathrm{~nm}(\mathrm{n}=6)$ but a secondary nucleation site was created right next to it increasing its width to $41 \pm 16 \mathrm{~nm}(\mathrm{n}=38)$. When a sharpened tip was used to minimize the effect of tip broadening, the width of the mature fiber was measured to be $13 \pm 4 \mathrm{~nm}(\mathrm{n}=14)$ which is similar to the width of other amyloids.[19] Assuming that the silk unit (GAGAGS) ${ }_{8}$ in SELP drives amyloid formation and one silk unit participates in one beta hairpin structure, the approximate width of amyloid which is determined by the half number of silk units is calculated to be $\sim 8.6 \mathrm{~nm}$ ( 24 residues $\times 0.36$ $\mathrm{nm} /$ residue). This is very close to the width measured using the sharpened tip and suggests that the silk unit plays a key role in amyloid assembly. The elastin units (GVGVP) may participate in beta sheet in a limited manner since the proline in the elastin unit tends to break the beta sheet structure.[49] The frequent secondary nucleation very close to a first nucleation site suggests that the one silk unit may be involved in forming two nucleation sites in its fully stretched conformation. 


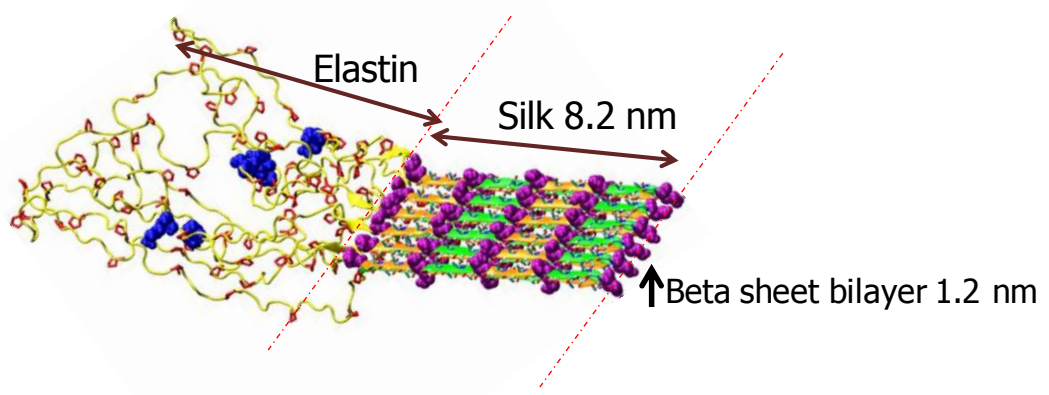

Figure 2-5. Speculated molecular conformation of amyloid nanofiber based on highresolution image and time-lapse nucleation experiment. The silk block folded into half of block width formed the core of nanofiber. It stacked in bata sheet bilayer manner resulting in stepwise height. The elastin block serves as an connection between two strands. 


\section{Chapter 3: Single-Molecule Force Spectroscopy of SELP}

\section{$\underline{3.1 \text { Introduction }}$}

Single-molecule force spectroscopy is a powerful method to study the mechanical properties of the polymer chain. By extending the polymer chain, the force response can be used to interpret the elasticity [50], secondary structure [51], as well as intermolecular interaction between chains $[52,53]$. In this section we utilized this method to study the mechanical properties of SELP protein adsorbed on the surface. It gained the insight on heterogeneity of the surface, as well as intermolecular interaction between the protein substrate and the AFM tip.

\section{$\underline{3.2 \text { Experimental Methods }}$}

Force Spectroscopy in normal direction was performed to compare the some experimental data with lateral force profile. Protein sample preparation follows the method explained previously. In summary, SELP (S815K) was deposited on mica to form fullycoated protein layer. This experiment was done in aqueous solution at room temperature. Molecular Force Probe-3D instrument (MFP-3D, Asylum Research, CA) was used with SiB probe on rectangular SiN soft cantilever (MLCT Bruker, CA) with a spring constant around 30pN/nm calibrated by thermal tuning method. The AFM tip was brought to the surface and subsequently pulled out in normal direction. The experiment was performed at a constant pulling rate of $10 \mu \mathrm{m} / \mathrm{s}$ over a distance of $500-1000 \mathrm{~nm}$. The normal force trigger point is 0.13 $0.27 \mathrm{nN}$. 


\subsection{Results}

When the tip was brought to the protein substrate, the approach curves showed longrange attractive force with the adhesive force $66 \pm 9 \mathrm{pN}$ over a range of $150 \mathrm{~nm}$. Once the tip is in contact with the substrate, it indented the surface around $10 \mathrm{~nm}$ before retracing from the substrate elastically. The adhesive well showed up with the magnitude of $135 \pm 14 \mathrm{pN}$ over a range of $150 \mathrm{~nm}$. We often observed the retrace curves that are well-known as the stretching of polymer chain according to Worm-Like-Chain model (WLC model). Generally, there were 2-5 peaks in retrace curves. There is no recognized spacing between peaks. Each peaks resulted in different persistent length and contour length. Therefore, we concluded that it is likely due to multiple peak stretching. In order to get single-molecule characteristic, nice curves were picked and the only last peaks that showed subsequence force dropped to zero were fitted with WLC model. The persistent length was $0.28 \pm 0.10 \mathrm{~nm}$, which is in a good agreement with persistent length of other proteins such as Bombyx mori silk-like [54], elastin-like [55], spider silk [52], or titin [51]. The contour length was $284 \pm 13 \mathrm{~nm}$ which was slightly less than calculated contour length of S815K molecule (320nm). Interestingly, among a hundred of force curves that we observed, there exhibited two retrace force curves that contour length was greater than single molecule length $(400 \mathrm{~nm})$. This indicated that the protein formed higher ordered structures such as dimer, which resulted in longer contour length. 


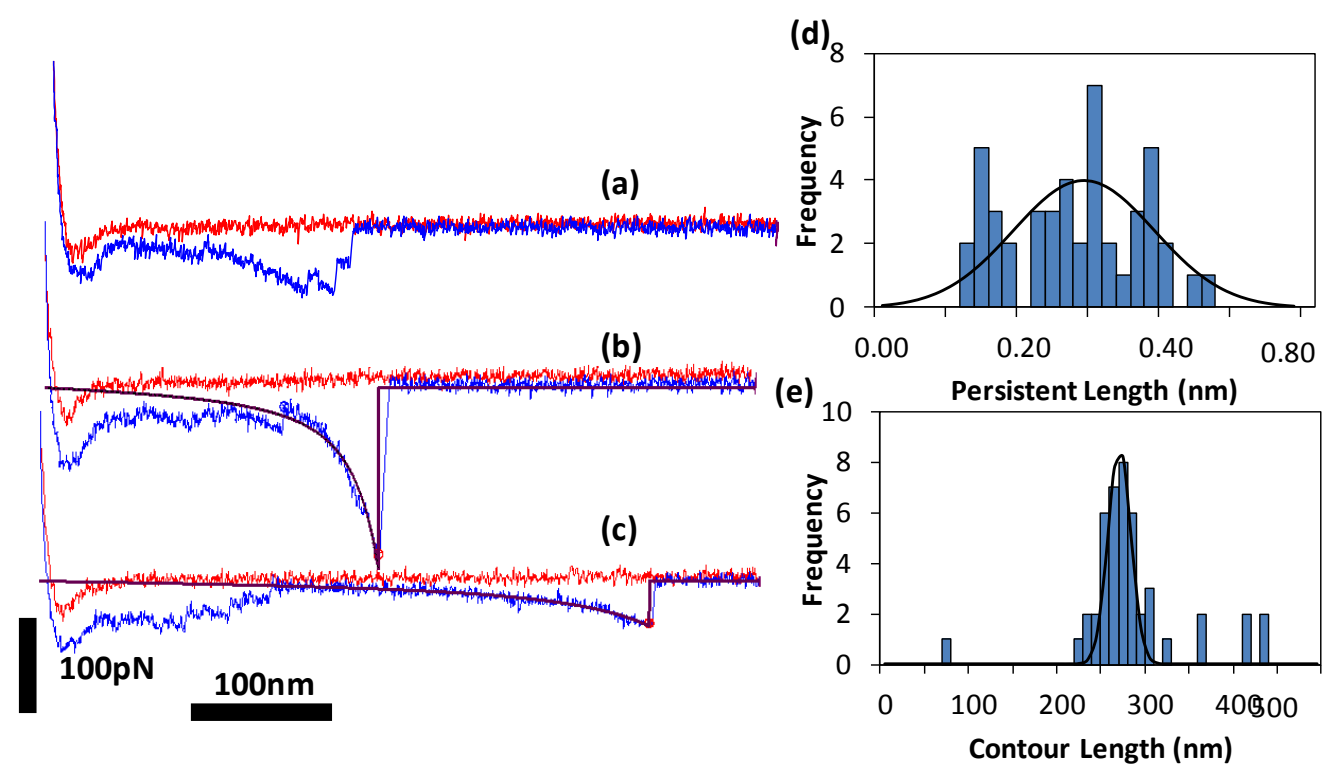

Figure3-1. Representative normal force spectroscopy curve obtained from the experiment. Red curve is approach and blue curve is retraction. (a) Major population of force curves consisted of multiple peaks indicated that there are multiple molecules attached to the tip. (b) Only force curves that clearly showed the last rupture peak are used to obtain the singlemolecule fitting parameters (fitting is shown in black line). In this case, $\mathrm{Lp}=0.27 \mathrm{~nm}$ and $\mathrm{Lc}=275 \mathrm{~nm}$. (c) Remarkable long force curve over 426nm. Attempting to fit with WLC model yields $L p=0.45 \mathrm{~nm}$ and $\mathrm{Lc}=552 \mathrm{~nm}$. (d) Histogram of the persistent length, (d) histogram of the contour length obtained from last peaks.

Now we are interested in the origin of adhesion hysteresis. There are two candidates for the long-range attractive force, either electrostatic or van der Waals [56]. While scanning, SELP on the surface was transferred to the SiN tip apex and created the coated layer of SELP on the SiN tip. The electrostatic originated from the interaction between positively charged SELP molecule on the surface ( $\mathrm{pI}=10)$ [40] and negatively charged SiN tip (pI=6). While van der Waals interaction arose from interaction between SELP substrate and SELP coating on the tip. To verify the hypothesis, we performed other experiment by varying the salt concentration of the solution (up to $0.5 \mathrm{M} \mathrm{NaCl}$ ). The result showed that as increasing the salt solution, the adhesion between protein and tip decreases, and it is completely disappeared at 
$0.05 \mathrm{mM} \mathrm{NaCl}$. This salt dependency supported that the adhesion hysteresis originated from electrostatic interaction.

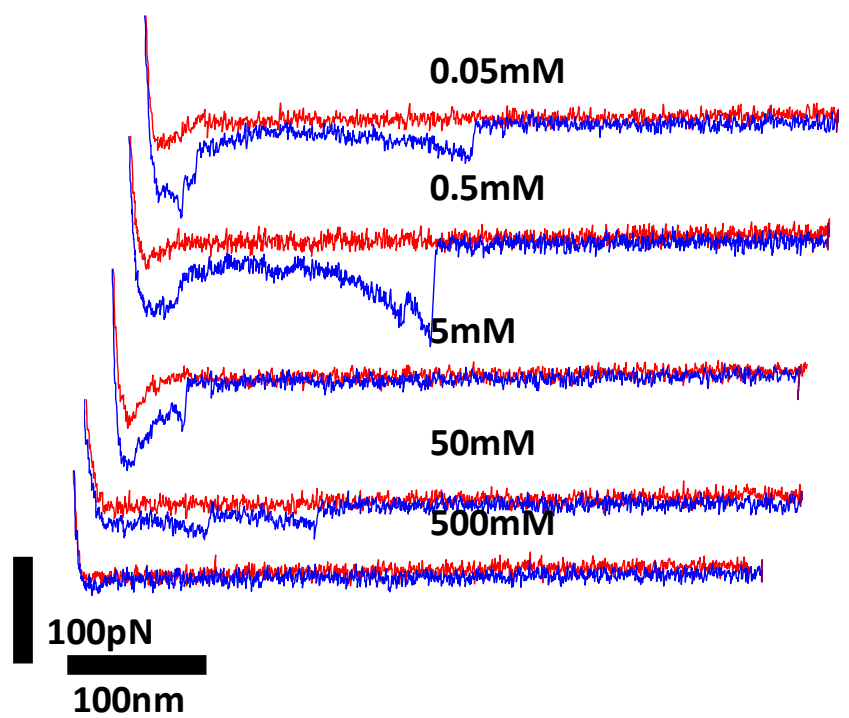

Figure 3-2. Representative normal force spectroscopy curves obtained at different $\mathrm{NaCl}$ concentration. The adhesion greatly decreases as increasing the salt concentration indicated that the electrostatic interaction involved in the adhesion force.

We fit the adhesion hysteresis region based on linear combination of electrostatic attraction between tip and substrate and compression of . [57] [56]Therefore, the force that tip subjected during approaching the surface is,

$$
F_{\text {approach }}(x)=\frac{4 \pi R \lambda \sigma_{R} \sigma_{s}}{\varepsilon} e^{-\frac{x}{\lambda}}-\frac{50 L k T}{s^{3}} e^{-\frac{2 \pi x}{L}}
$$

Where $\mathrm{F}(\mathrm{x})$ is total adhesion force exerted on the tip at separation distance $\mathrm{x}$ from the surface. $R$ is radius of the tip, $\lambda$ is Debye's screening length, $\boldsymbol{\sigma}_{\mathbf{R}}$ and $\boldsymbol{\sigma}_{\mathbf{s}}$ correspond to surface charge density of tip and substrate respectively. $\varepsilon$ is dielectric of the medium. $\mathrm{L}$ is the thickness of polymer brush film, $\mathrm{kT}$ is thermal energy, and $\mathrm{s}$ is mean spacing distance between polymer chain. 
When the tip indented the protein film until deflection reached set point, the tip retraced from the surface, and it subjected to JKR, electrostatic, in addition with WLC model.

$$
F_{\text {retrace }}(x)=\frac{4 \pi R \lambda \sigma_{R} \sigma_{s}}{\varepsilon} e^{-\frac{x}{\lambda}}-\frac{50 L k T}{s^{3}} e^{-\frac{2 \pi x}{L}}+f_{w l c}(x)
$$

Where,

$$
f_{w l c}(x)-F_{0}=\frac{k_{b} T}{L_{p}}\left[\frac{1}{4\left(1-\frac{x}{L_{c}}\right)^{2}}\right]-\frac{1}{4}+\frac{x}{L_{c}}
$$

Table 3-1 Fitting parameters in force-extension curve from SELP protein film

\begin{tabular}{ll|ccccc}
\hline tors & fac & 's & $\begin{array}{c}\text { Debye } \\
\text { Length }\end{array}$ & $\begin{array}{c}\text { Prefactor in } \\
\text { electrostatic term }(\mathbf{p N})\end{array}$ & $\begin{array}{c}\text { Film's } \\
\text { thickness }(\mathbf{n m})\end{array}$ & \multicolumn{2}{c}{$\begin{array}{c}\text { Spacing } \\
\text { between } \\
(\mathbf{n m})\end{array}$} & polymer \\
\hline \multirow{2}{*}{ ace } & $\mathbf{T r}$ & 8.29 & 3392 & 50.4 & 0.68 \\
race & $\mathbf{R e t}$ & & 9.30 & 2630 & 54.6 & 0.61 \\
\hline
\end{tabular}

\subsection{Discussions}

Film's thickness is high because the SELP is transferred to the tip and coated the tip, result in large effective tip radius and effective polymer's film thickness that undergoes compression. Prefactor in electrostatic term is left unsolved since there are multiple variable that are unknown. Further research is required. After pulling experiment, the area scan was performed to look at the topology of the film. There is no newly-formed nanofiber nor change in the topology indicated that this protein film pursues self-healing effect and simply just pulling the protein chain didn't result in the nucleation formation. 


\section{Chapter 4: Lateral Force Microscopy of SELP}

\subsection{Introduction}

LFM can measure the frictional force in the scan direction by observing the twisting deflection of the cantilever. The twisting of the cantilever is caused by both chemical and mechanical interactions between the moving tip and the substrate

\subsection{Experimental Methods}

SELP sample was prepared in the same manner as explained previously. LFM experiments were carried out on a Molecular Force Probe-3D instrument (MFP-3D, Asylum Research, CA). A rectangular SiN probe (MLCT Bruker, CA) was used. The spring constant in the normal direction was calculated using the MFP-3D software (Igor Pro Version 6.22A, MFP3D) using the thermal tuning method, which gave a value of $30 \mathrm{pN} / \mathrm{nm}$. The lateral sensitivity was calibrated right before each experiment based on the method explained by Ogletree, et.al. [58] and Varenberg, et.al. [59]. The lateral sensitivity values varied between $100-250 \mathrm{pN} / \mathrm{mV}$, deviating from batch-to-batch of the cantilever, location of the laser spot on the cantilever and location of the chip. Data were acquired at 516 points/ $\mu \mathrm{m}$. The normal force set point was $0.14 \mathrm{pN}$. We used the $140-\mathrm{pN}$ normal force since it is the smallest force that is reliable considering the stiffness of the tip and resolution of AFM (0.1V deflection). During the scan, SELP was also attached to the AFM tip by physisorption in lateral scanning mode resulting in greater effective tip radius as well as greater contact between tip and the substrate.

Height profiles were the result of the tip response to normal forces while lateral profiles were the result of tip response to forces parallel to the scan direction. During trace, 
increases in friction result in positive lateral force signals while during retrace it results in negative lateral force signals.

Time-lapse experiments were carried out in "slow-scan disabled" mode. The height and friction images were obtained as a stack of topology profiles in a single line trajectory as a function of time. The time-lapse experiments were performed for $128 \mathrm{sec}$ or 512 total scan lines per each experiment. The scan frequency was $2 \mathrm{~Hz}$, thus the spatial resolution was 0.25 seconds per line. After completion of each time-lapse frame, an area scan was obtained in the same location to verify that the nucleation site leads indeed to nanofiber elongation from that location, and that it was perpendicular to the scan direction.

\subsection{Data Analysis:}

In time-lapse experiments, we defined nucleation by a localized height increase in the background of at least $0.5 \mathrm{~nm}$, which was stable after a few seconds. The lateral force profiles were fitted using a worm-like chain model and an exponential function using the leastsquares likelihood estimation method in a IgorPro software (Wavemetrics, USA).

\subsection{Increase in Lateral Force Results in Amyloid Nucleation}

The background frictional force between the SELP modified tip and SELP adsorbed mica was $165 \pm 89 \mathrm{pN}(\mathrm{n}=12)$. Figures $4-1 \mathrm{a}$ and $4-1 \mathrm{~b}$ show a height image and a lateral force image of a newly formed nucleus obtained using time-lapse LFM. The height profile shows that there was a new nucleation site formed at $36.5 \mathrm{sec}$ (Figure 4-1a). Figure 4-1b shows the variation of lateral force along the same scan line as a function of time. A newly formed nucleus is also visible in the lateral force image since lateral force slightly increases momentarily when the tip contacts a nucleus from the side. Notably significant increase in lateral force was observed when a new nucleation site was formed. Figure 4-1c shows three 
consecutive line scan profiles of height and lateral force at three different points in time. At $36 \mathrm{sec}$, the tip moved laterally from left to right and did not show any significant variations in either height or lateral force profile. In the following scan from right to left, the lateral force significantly increased by $\sim 0.8 \mathrm{nN}$ and suddenly dropped, while no change in height was observed. In the subsequent scan, a sudden increase in height in the marked location was observed, indicating the formation of a new nucleation site while the lateral force profile remained at a constant level. Not all the increased frictional force profiles resulted in the formation of nucleation sites. However, whenever new nucleation sites were formed, the increase in lateral force always preceded nucleation, which indicates that an increase in frictional force is a necessary condition for a nucleus formation.

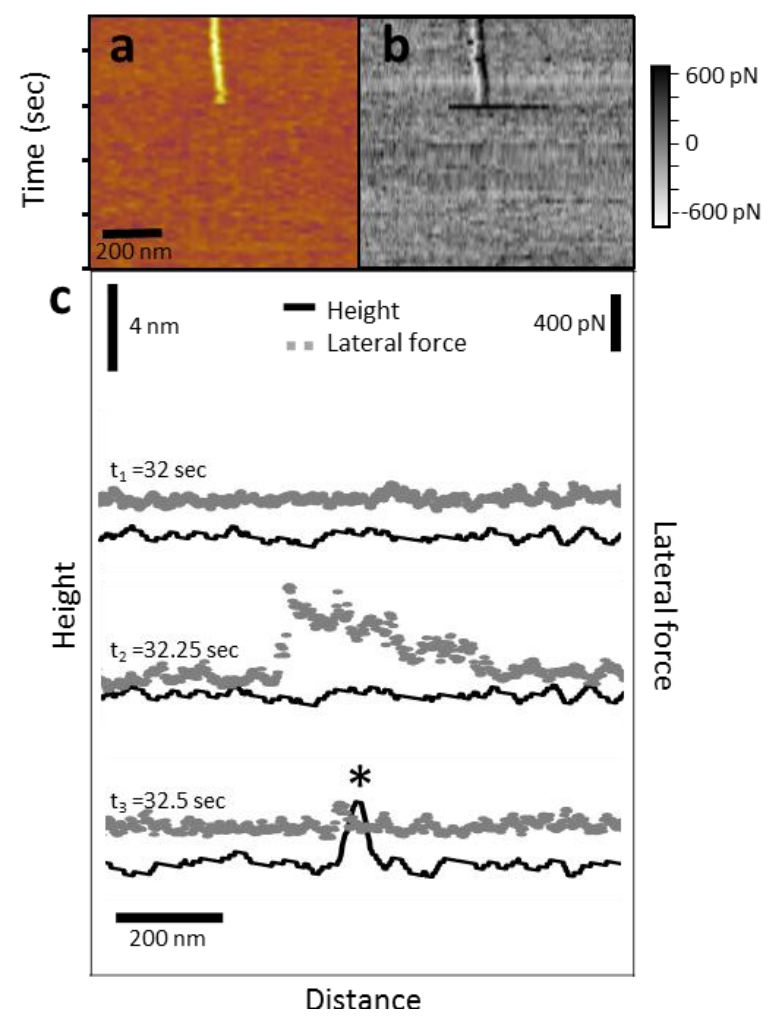

Figure 4-1. (a) A height image showing a single nucleation site formation during a time-lapse LFM measurement, (b) a lateral force image showing increased lateral force right before a new nucleation event, (c) three consecutive height and lateral force profiles during a nucleation. (height in black and lateral force in grey) A sudden increase in the lateral force profile led to a height increase in the following scan indicating that a new nucleation site $(*)$ was formed. The black arrow shows the scan direction. 


\subsection{Increase in Lateral Force Originates From Stretching Laterally}

We analyzed $~ 100$ lateral (frictional) force profiles that lead to nucleation. We classified them into three categories based upon their shapes: a worm-like chain (WLC) profile, a force profile with a sawtooth pattern and an exponential profile. The worm-like chain profile is shown in Figure 4-1a. The frictional force increased nonlinearly and suddenly dropped to the background frictional force level after reaching a maximum force. The nonlinearly increasing portion was fitted using the WLC model as shown below.

$$
F(x)=\frac{k_{b} T}{L_{p}}\left(\left[\frac{1}{4\left(1-\frac{x}{L_{c}}\right)^{2}}\right]-\frac{1}{4}+\frac{x}{L_{c}}\right)
$$

Where $\mathrm{F}$ is a lateral force along the stretching direction, $\mathrm{k}_{\mathrm{b}}$ is Boltzmann constant, $\mathrm{T}$ is the absolute temperature, $\mathrm{L}_{c}$ is a contour length and $\mathrm{L}_{\mathrm{p}}$ is a persistence length. When the nonlinear profile was fitted with the WLC model, the persistence length was $0.25 \pm 0.2 \mathrm{~nm}$ $(n=15)$ and the contour length was $332 \pm 161 \mathrm{~nm}(\mathrm{n}=15)$. This persistence length matched well the persistence length of a elastin molecule measured from single molecule stretching experiments[60],[61], implying that the stretching behavior of SELP is mainly determined by the elastin units in SELP. The fitted contour length was comparable to its calculated contour length of $295 \mathrm{~nm}(=818$ residues $\times 0.36 \mathrm{~nm} /$ residue $)$, indicating that a single SELP molecule is stretched along the scan direction during this process. The persistence length agrees well with the value $(0.28 \pm 0.1 \mathrm{~nm}(\mathrm{n}=50))$ measured from normal force pulling as well. The maximum stretching force level of the WLC profiles was $318 \pm 128(n=15)$. The fact that the frictional force profile fitted well with the WLC model strongly suggests that a single SELP molecule was stretched when a lateral force increased. 


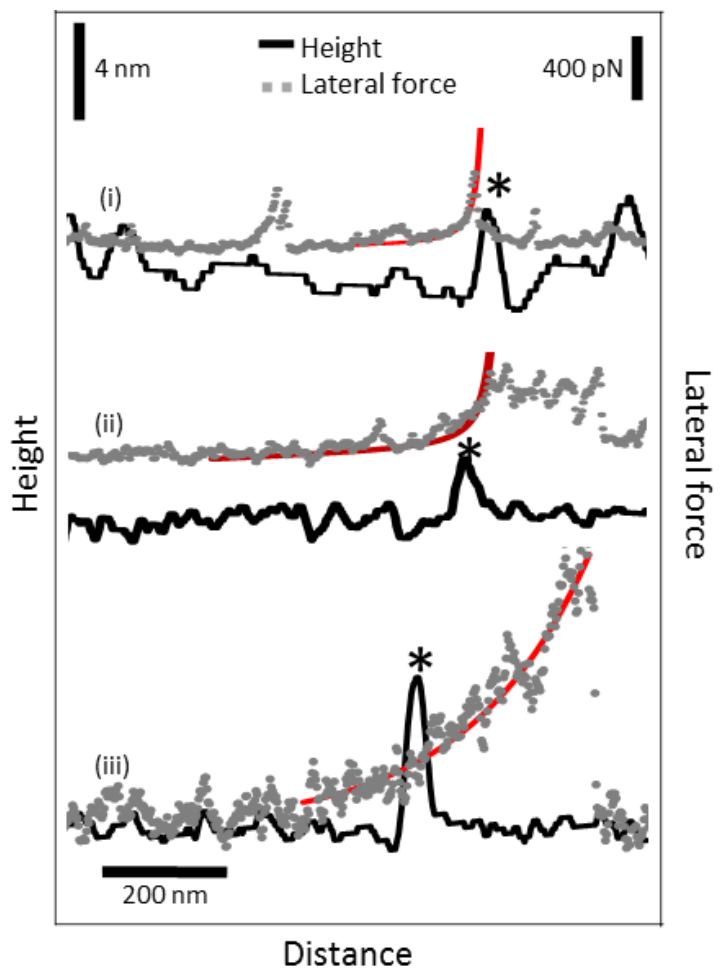

Figure 4-2. Three types of frictional force profiles which resulted in nucleation during timelapse LFM measurements. (a) Worm like chain (WLC) profile. The WLC model fit is shown in red. (b) Force profile with a sawtooth pattern. (c) Exponential force profile.

The second type of a lateral force profile shows a nonlinear force increase followed by a sawtooth pattern (Figure 4-2b). The nonlinear portion of the profile was fitted with the WLC model. The persistence length and the contour length were $0.21 \pm 0.2 \mathrm{~nm}(\mathrm{n}=55)$ and $314 \pm 95 \mathrm{~nm}(\mathrm{n}=55)$, respectively. The average peak force in the sawtooth pattern was $218 \pm 158 \mathrm{pN}$ ( $\mathrm{n}=55$ ) varying from $133 \mathrm{pN}$ to $691 \mathrm{pN}$. The average peak to peak distance was $33 \pm 9 \mathrm{~nm}(\mathrm{n}=75)$ with the number of peaks in one pattern varying from 2 to 14 . The stretching length which is the total stretched length of the molecule was $341 \pm 201 \mathrm{~nm}$, again which is close to the contour length of SELP, suggesting that a single SELP molecule is stretched when the lateral force increased. The narrow distribution of peak to peak distances suggests that this sawtooth pattern is probably due to the unraveling of well-defined structures. 
Multiple molecule attachments and detachments can produce a similar profile. However based on the range of maximum stretching forces and the observation that the last peak that is usually the one with the highest stretching force, this profile is most likely due to stretching one single SELP molecule or a few SELP molecules that are connected in series. Hence we speculate that the sawtooth pattern originates from stretching a single SELP molecule followed by disruption of locally ordered structures in a serial manner. The sawtooth pattern was previously observed when beta sheet secondary structures on the surface was disrupted by mechanical force [62]. In that study, the unfolding force ranged from 70 to $200 \mathrm{pN}$, in a good agreement with our results. A sawtooth pattern indicates the disruption of ordered structures such as beta hairpin, exposing previously hidden length and thus reducing the force level. If the silk unit participates in locally ordered structures and the elastin unit is excluded from these ordered structures, the extension length due to the disruption of the ordered structures is largely determined by the elastin unit. The extension due to the elastin unit is calculated to be $\sim 29 \mathrm{~nm}$ ( $=80$ residues $\times 0.36 \mathrm{~nm} /$ residue $)$. This calculated length is very close to the experimentally observed peak to peak distance, implying that the sawtooth pattern is due to the consecutive disruption of locally ordered structures composed of the silk unit.

The exponential profile was fitted using the following equation [63].

$$
\mathrm{F}(\mathrm{x})=\mathrm{F}_{\mathrm{p}} \exp \left(\frac{\mathrm{x}-\mathrm{x}_{\mathrm{p}}}{\mathrm{D}}\right)
$$

Where $F_{p}$ is a peak force, $x_{p}$ is a location of a peak force and D is a scaling length. The scaling length is the characteristic distance of the molecular network system. When an exponential profile was fitted to the WLC model, it produced an order of magnitude smaller persistence length $(0.08 \pm 0.28 \mathrm{~nm}(\mathrm{n}=32))$ and a long persistence length $(512 \pm 241 \mathrm{~nm}(\mathrm{n}=32))$, which clearly indicates that this profile cannot be fitted using the WLC model. The maximum stretching force was $1011 \pm 970 \mathrm{~nm}(\mathrm{n}=32)$ which was significantly higher than the WLC profile or the profile with the sawtooth pattern. The higher maximum stretching force and 
unusually small persistence length indicates that multiple molecules are involved during stretching. The exponential model has been used to describe the mechanical behavior of molecular and supramolecular network structures of the flagelliform protein of spider silk.[52] Flagelliform protein is rich in proline and contains highly repetitive beta-spiral sequences which are similar to the elastin unit of SELP. We speculate that our exponential profile originates from molecular networks where multiple SELPs are interacting via inter-and intramolecular interactions. The crosslinks are likely formed by silk units and elastin units connect those physical crosslinks. The molecular network seems to be mechanically robust since we did not observe any significant drops in force during stretching which would indicate a release of individual chains. The stretching length was $482 \pm 271 \mathrm{~nm}(\mathrm{n}=32)$ varying widely from $152 \mathrm{~nm}$ to $1029 \mathrm{~nm}$. A significant portion of the stretching lengths measured was longer than SELP's contour length, confirming that multiple molecules are participating. The scaling length obtained from the fitting was $127 \pm 55 \mathrm{~nm}(\mathrm{n}=32)$. The scaling length is a parameter used to relate the distance between crosslinking points in the network structure. The long stretching length and the significantly high stretching force observed strongly suggest that an oligomeric network state of SELP is responsible for the exponential force behavior during stretching. 


\subsection{Stretching Force Dependency on Nucleation Formation}

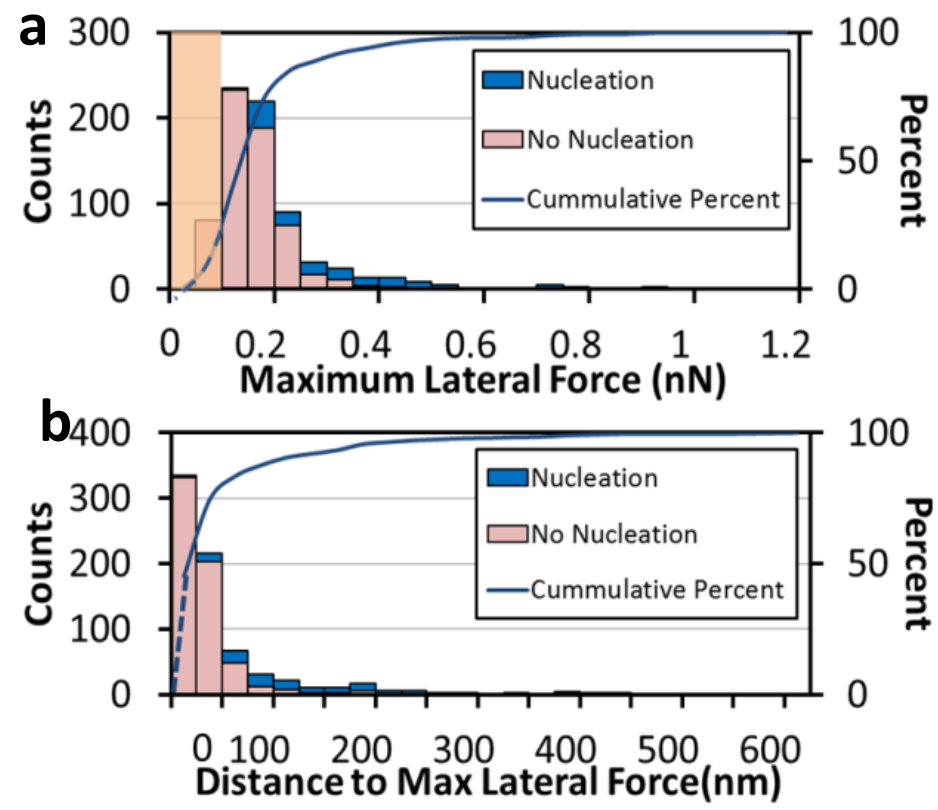

Figure 4-3. (a) histogram of maximum lateral force, (b) histogram of stretching length

The probability of forming a nucleation site was significantly dependent on the shape of the force profiles. Among all the WLC profiles that we analyzed, only a fraction $(\sim 5 \%)$ of them successfully formed nucleation sites. This observation is not surprising since nucleation requires multiple molecule interactions. WLC behavior still led to nucleation in some cases probably due to surface diffusion of other chains or local ordering of the single chain after detachment. On the contrary, a plateau profile with the sawtooth pattern or an exponential profile showed $18 \%$ and $63 \%$ probability of forming a nucleation site respectively. The increased probability of nucleation formation for a profile with a sawtooth pattern shows that local ordering of SELP is crucial for nucleation. Even though the local ordering is disrupted during stretching it is expected to reform quickly as the chain is detached from the tip and allowed to relax[64]. The exponential profile showed significantly increased success rate of nucleation. This is due to aligning multiple molecules by stretching the oligomeric SELP network along the scanning direction. Stretching induces more ordering which will enhance 
nucleation. Keten et al. showed that the number of hydrogen bonding between beta sheets increased as a nanocrystal of beta sheet was stretched in molecular dynamic simulations [65]. Mechanical force helps to overcome the energy barrier of kinetically trapped states, facilitating the formation of locally ordered structures, hence nucleation[65].

\section{$\underline{4.7 \text { Discussion }}$}

It was proposed that the lateral force originated from instantaneous bond forming/breaking (chemical friction) between the tip and the substrate[66]. In polymer films, including protein, Overney et al. described the friction due to stretching the protruded chain segment, which causes sawtooth patterns in lateral profiles[67]. Stretching of the long molecules is a major origin of friction especially for elastomers [32, 37], synthetic polymers [68] and various protein films[35, 36]. Plowing [69] and dragging protein chains on the surface [70] can also increase the frictional force.

The frictional force profiles fitted with the WLC model shows that SELP with these profiles is largely unstructured like the PEVK region in titin [71]. It also demonstrates that a single SELP molecule can form a nucleus but its success rate is very low. The fact that the locations of the nuclei are not always correlated with the origins of the stretching indicates that the nucleus can form in any part of the stretched molecules. The force profile with the sawtooth pattern indicated that weak secondary bonds, also called sacrificial bonds, in the locally ordered structures break during stretching[72]. These sacrificial bonds can reform reversibly, thereby providing a "self-healing" nature to the material. The strength of the bond and the periodicity of the peaks indicate that the partially ordered silk units are likely to be responsible for this behavior. Additionally, the significantly higher chance of forming nucleation of these force profiles indicates that the sacrificial bonds broken during stretching quickly rearrange to form a beta hairpin structure which can accelerate nucleus formation. 
The last peak of the sawtooth pattern was not different from the other peaks indicating that SELP is attached to the AFM tip with a similar force level as the force required for disrupting secondary structures.

The exponential force profiles showed high probability (63\%) of nucleation. The exponential force profile was observed in both capture-silk molecules and intact strands of capture silk [52]. Considering that its maximum stretching force is significantly higher than those of other force profiles, it is clear that some aggregated structure is interacting with the SELP modified AFM tip with higher adhesion force. High resolution in situ AFM studies of self-assembly dynamics revealed multiple oligomeric species involved in dynamic assembly and disassembly processes on surfaces[46]. We speculate that the different force profiles may indicate the heterogeneous populations of SELPs on the surface. Hence an exponential force profile may result from oligomeric states interacting with the SELP modified tip, exhibiting long stretching lengths as well as high maximum stretching forces. In this case, multiple molecules in the network are expected to be aligned along the stretching direction, which will have a significant advantage of forming nuclei via multiple intermolecular interactions in an extended conformation. Molecular dynamics simulation shows that stretching induces more hydrogen bonding, which in turn induces the formation of beta-sheet structures in the amorphous region[65]. We speculate that molecular stretching induces the ordering of the SELP backbones which can serve as a template for further nucleation.

Figure $4 \mathrm{~d}$ shows a plot of maximum stretching force vs. stretching distance of all the force profiles that resulted in nucleation. The maximum stretching force of the WLC profiles varied from $120 \mathrm{pN}$ to $600 \mathrm{pN}$ and most of their stretching lengths were shorter than the calculated contour length of SELP. A force profile with the sawtooth pattern showed a similar range of maximum stretching force but $\sim 30 \%$ of the data showed longer contour lengths than the calculated contour length of SELP, indicating that dimeric or trimeric molecules might have formed. The maximum stretching force for the exponential profile ranged from 335 to 
$2000 \mathrm{pN}$ and its stretching length varied widely from $150 \mathrm{~nm}$ to $1 \mu \mathrm{m}$, well beyond the contour length of a single SELP molecule. This indicates that an exponential force profile is likely to originate from multiple SELP interactions between the SELP modified tip and the SELP coated mica. The oligomeric SELP is formed by intermolecular interactions that lead to the formation of a molecular network structure. When the SELP modified tip interacts with the oligomeric SELP on mica, the adhesion force is expected to be higher, resulting in a higher maximum stretching force and longer stretching length. McAllister et al. showed that proteins in the aggregating state exhibit larger adhesion forces than those in the nonaggregating state. [73]

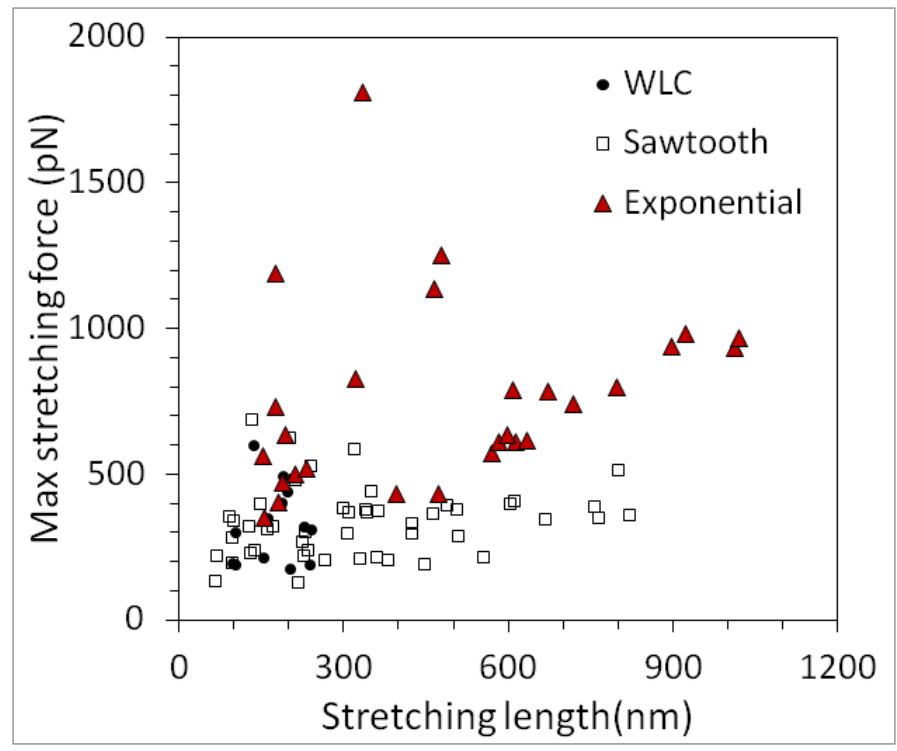

Figure 4-4. Plot of maximum stretching force vs. stretching distance.

We believe the silk block is mainly responsible for the kinetically trapped states. SELP-415K which contains 4 blocks of the silk unit formed nanofibers instantaneously, whereas it took days for SELP-815K containing 8 blocks of silk unit to grow on a mica surface[40,41]. This clearly indicates that the silk content has significant effect on the rate of amyloid nucleation on the surface. Another key role of the stretching force is that it aligns the 
backbone of the SELP along the scanning direction, creating a template which enhances the growth of the nanofiber perpendicular to the scanning direction.

Recently, it was found that the moderate shear applied can trigger the coil-to-beta sheet transitions presumably by stretching the macromolecule before folding it into a parallel beta-sheet.[74, 75]. Our results strongly suggest that macroscopic shear force or sonication may play a similar role of stretching as observed in our system, providing an extended conformational state, a template for further nucleation growth and thus amyloid assembly.

\subsection{Speculation on mechanisms of nucleation formation under nanomechanical} stretching

During incubation, the SELP molecule will adsorb on the mica surface via a flycasting mechanism due to electrostatic attraction. The adsorbed SELP is likely to have a reduced number of states accessible to the unfolded state, thereby reducing the entropic cost of folding on the surface compared to the bulk state. There could be limited surface diffusion of SELP locally, forming oligomerized states. However, the high activation energy of the partially ordered structure of the silk unit slows down the nucleation process significantly. When the SELP was mechanically stretched by the AFM tip, the extended conformation causes other molecules to assemble onto it, forming a nucleus (Figure 4-5). The laterally stretched molecules will survey the protein-coated surface. The extended conformation provides an ideal template for nucleation. Nearby silk units will bind to the template to form a critical size for nucleation. Inter- or intramolecular interactions between multiple SELPs stretched together or local fluctuations of conformational states cause growth of assembled nuclei through an induced fit mechanism using a template formed during stretching [76].

Once the nucleus reaches the critical size, its lifetime is long enough for other molecules to self-assemble into amyloid fibrils. During stretching the backbone of SELP is aligned along 
the scan direction, which further guides the assembling direction of amyloid [45]. It is also possible that additional ordered structures may align during stretching, guiding the directions of amyloid growth.

During the mechanical relaxation process, the SELP can reconfigure itself and form a hairpin unit. Amyloid with $\sim 8 \mathrm{~nm}$ width is formed when one silk unit folds onto itself. After SELP is detached from the tip, it may further accelerate nucleation by interacting with aligned oligomeric states during stretching. This stretching process may mimic a system in living organisms where the regulatory protein chaperone reverses misfolded protein intermediates to their native state by stretching.[77]

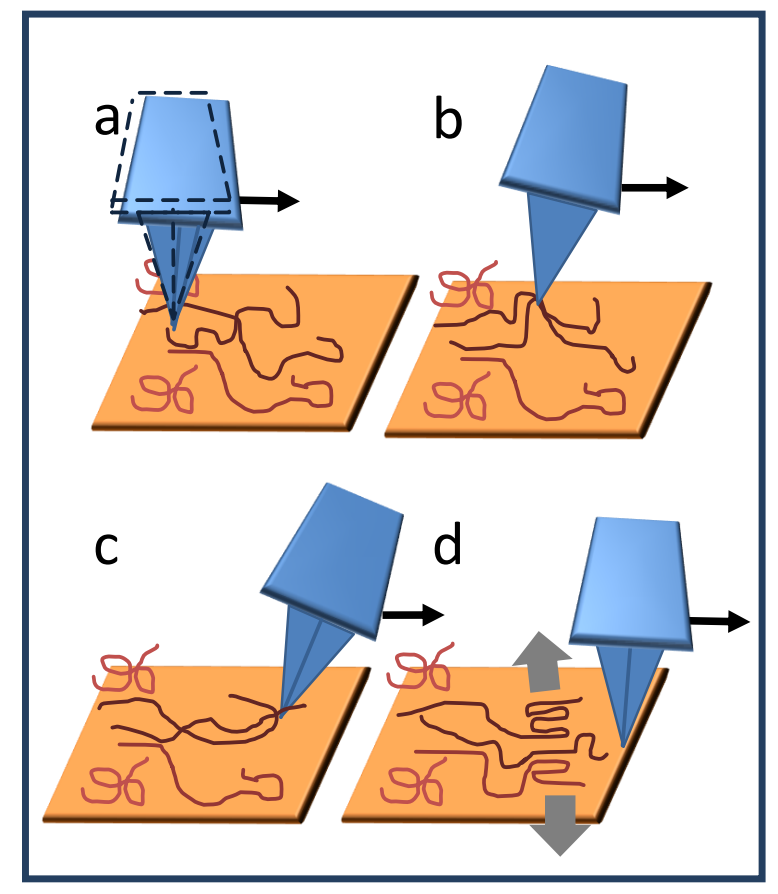

Figure 4-5. Speculated molecular mechanism of nanomechanically induced amyloid nucleation. (a) SELP on the surface attached to SELP modified tip via nonspecific interactions. (b) As the tip moves along the scan direction, SELP is stretched, aligning its backbone along the scan direction. This nanomechanical stretching leads to an increase in lateral force. (c) The extended conformation of SELP may serve as a template for further nucleation assembly by incorporating nearby SELP molecules. It also aligns small segmented secondary structures along the scan direction. (d) SELP detaches from the tip. This relaxation process may further enhance secondary structure formation, aiding a hierarchical assembly of nucleation. 


\section{Chapter 5: Exploration of the Activation Energy of Mechanically induced Amyloid Nucleation}

\subsection{Introduction}

In this chapter, we will explain the model of mechanical force from AFM tip induced amyloid nucleation. By varying the normal force set point, the nucleation rate was determined. The nucleation rate was used to obtain the statistical thermodynamics parameter so called the activation energy barrier. This chapter will elucidate the stoichiometric nature of this process and serve as the guideline for future amyloid nucleation model.

\subsection{Theory}

When the solution is incubated on mica, silk-elastinlike peptide (SELP) absorbs on muscovite mica and forms densely packed protein layer. In contact mode, AFM tip approaches the substrate and get in proximity contact with the SELP precursor. A bond between tip and protein forms, leads to interaction between tip and protein chain. During the scan, the lateral force profile indicates that protein chains are picked up by the AFM tip and stretched along the scan direction while the normal force remains constant by the feedback loop of the AFM. As the tip travels away from the pick-up location, SELP molecule is subjected to the tension in the chain and as a consequence, the protein is extended along the scan direction in a high enthalpic state. At the maximum rupture force, the SELP chain is released from the tip and refolds, lead to SELP fiber formation on the mica surface.

It is the complicated processes involving non-specific interaction between protein film and the tip, the adhesion of protein on surface and the tension in the protein chain that serves as the nuclei for the formation of beta sheet. Interestingly, those three force (energy) 
components need to be balanced unless protein couldn't successfully extend and neighboring protein cannot diffuse to self-assemble into a nanofiber.

Since there are multiple processes involved in mechanical force induced amyloid nucleation, the protein needs to overcome multiple energy barriers. While the normal force exerted by the AFM tip increases the frictional force, it also increases the rate of nucleation. This effect can be explained by the fact that the greater the frictional force, the greater chance that the protein will be picked-up by the AFM tip and stretched, resulting in nucleation.

Here, we attempt to obtain the effect of mechanical force from the AFM tip on the amyloid formation under the assumption that the normal force applied form the tip enhances the nucleation rate by increasing the chance of bond formation between tip and substrate protein. Using beautiful transition state theory derived by Kramer [1], the probability that the nucleation reaction will succeed is inversely proportional to the exponent of the free energy of activation of the reaction.

$$
p \sim \exp \left(-\frac{\Delta G^{\ddagger}}{k_{b} T}\right)
$$

The exponential term corresponds to the Boltzmann-Arrhenius exponential factor in the rate of reaction equation.

In the presence of compression from tip, the energy dissipated between tip and sample lowers the activation barrier hence increasing the probability of a bond formation.

$$
p(f) \sim \exp \left(-\frac{\left(\Delta G^{\ddagger}-f x\right)}{k_{b} T}\right) \sim \exp \left(\frac{\left.-\Delta G^{\ddagger}+f x\right)}{k_{b} T}\right)
$$

Where $f$ is the compressive force, $x$ is the characteristic length related to the width of the activation barrier in the reaction coordinate.

Also, the normalization with probability of no nuclei formation is required, 


$$
P(f)=\frac{\exp \left(\frac{\left.-\Delta G^{\ddagger}+f x\right)}{k_{b} T}\right)}{1+\exp \left(\frac{\left.-\Delta G^{\ddagger}+f x\right)}{k_{b} T}\right)}
$$

Finally we arrived at the probability of successful stretching as a function of applied compressive force,

$$
P(f)=\frac{\mathrm{B} * \exp \left(\frac{f}{f_{0}}\right)}{1+\mathrm{B} * \exp \left(\frac{f}{f_{0}}\right)}
$$

Where,

$$
B=\exp \left(\frac{-\Delta G^{\ddagger}}{k_{b} T}\right)
$$

And,

$$
f_{0}=k_{b} T / x
$$

\subsection{Experimental Methods}

The sample preparation and experimental setup followed the methods explained previously. SiN tips with different spring constant were used to confirm the independency of tip's stiffness. The normal force varied from $0.35 \mathrm{nN}$ to $2.8 \mathrm{nN}$. At higher normal force level, the effect of plowing can be seen due to the removal of protein from the surface. To study the frictional force on the surface, lateral force microscopy was performed.

\subsection{Time-Lapse Experiment: Data interpretation}

The above model is applied for time lapse nucleation experiment, where the tip is scanned back and forth on the same molecules during single line scanning. When tip travels over a SELP monomer, bond(s) between tip and SELP start to form. Sufficient number of bonds is required for successful stretching and nucleation. The tip might not form bonds 
strong enough to tolerate the rupture of SELP and the fiber cannot be formed. If the bonding is strong enough, the SELP is ruptured and nucleation is observed right in the next scan. We simplify the model by assuming that there is no memory effect on the protein after unsuccessful stretching, meaning that protein recovers to the original conformation after detachment. This hypothesis is widely used in modeling protein folding and is known as the two-state model. [78-80]. The probability to observe the nucleation after $\mathrm{t}^{\text {th }}$ scan is then equal to the probability that the tip will successfully stretch the protein after $t^{\text {th }}$ trials. This probability follows a geometric distribution.

$$
\operatorname{Pr}(t, f)=P(f)[1-P(f)]^{t-1}=\left[B \exp \left(\frac{f}{f_{0}}\right)\right]\left[1-B \exp \left(\frac{f}{f_{0}}\right)\right]^{n-1}
$$

\subsection{Normal Force Dependency on Amyloid Nucleation}

In the time lapse line scan experiment, the tip is drawn on the SELP molecule with afrequency of $1 \mathrm{~Hz}$. Thus there are two attempts (trace and retrace) in every one second. The number of attempt required to observe the nucleation is plotted as function of theapplied force, $f$.

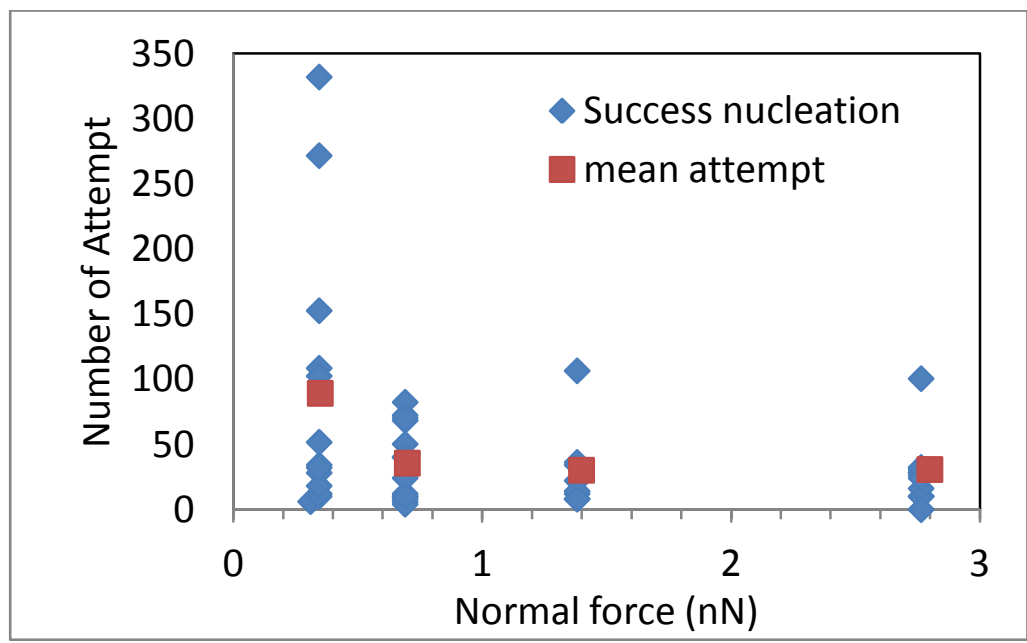

Figure 5-1. Number of scan until the nucleation site is observed is proportional to the normal force set point. At higher normal force, the nucleation site tends to form faster, resulting low number of attempted scans. 
In order to obtain the probability of nucleation at different normal force, the arithmetic mean of the number of attempts $[t(f)]$ is found and relate to the probability of nucleation $\operatorname{Pr}(f)$ via the equation,

$$
P(f)=\frac{1}{[t(f)]}
$$

Table 5-1. Probability of Nucleation at different normal force

\begin{tabular}{c|cccc}
\hline Normal force (nN) & $\mathbf{0 . 3 5}$ & $\mathbf{0 . 7}$ & $\mathbf{1 . 4}$ & $\mathbf{2 . 8}$ \\
\hline Probability & 0.011 & 0.028 & 0.033 & 0.033 \\
\hline
\end{tabular}

Finally, the probability of nucleation can be used to calculate the activation energy barrier in which $B=0.032 \pm 0.009$ and $f_{0}=0.60 \pm 0.9 \mathrm{nN}$. This value corresponds to the activation energy of $3.4 k_{b} T$ or $2 \mathrm{kCal} / \mathrm{mol}$, which is around one hydrogen bond.

\subsection{Normal Force, Frictional Force, and Amyloid Nucleation Rate}

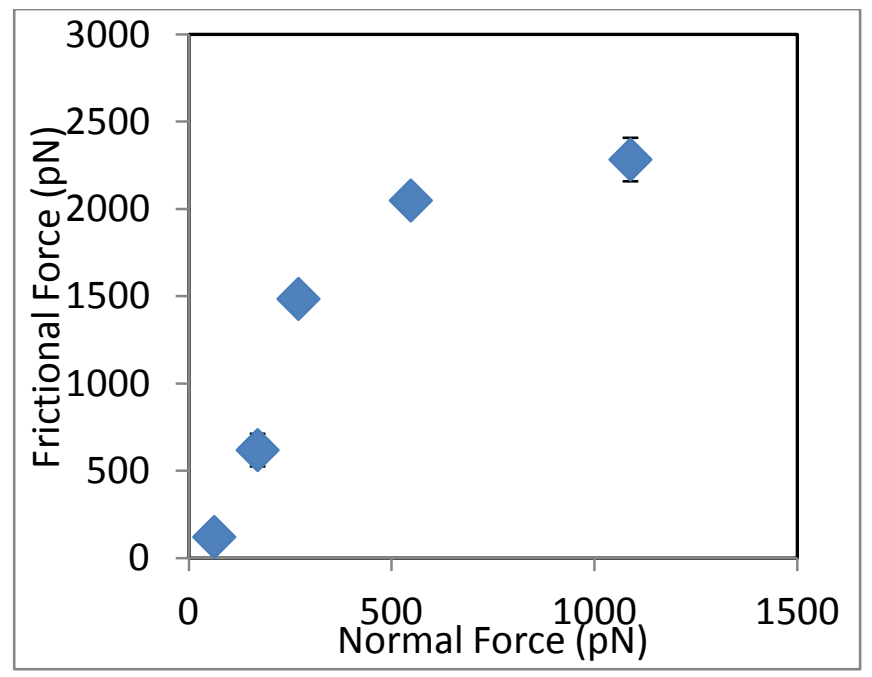

Figure 5-2. Relationship between normal force and frictional force did not follow AmontonsCoulomb law, which is the linear relationship. Some data showed the standard deviation bar smaller than the data points.

When the normal force was varied to observe the frictional force change, the dependency didn't follow linear relationship, instead, it followed the exponential behavior. 
When the tip is in contact with the substrate, increasing the normal force will greatly increase the contact area resulting in greater numbers of bond formation between protein and the tip, hence greater frictional force. Number of bonds formed between tip and protein film is exponentially increased with normal force, so called kinetics rate equation [32]. This result supported the above theory that the normal force affected the amyloid nucleation by increasing the number of bond formation and resulting in more pick-up probability. 


\section{Chapter 6: Single Nanofiber Patterning}

\section{$\underline{6.1 \text { Introduction }}$}

Understanding the molecular mechanism of mechanical force induced self-assembly of SELP nanofiber served as the model system to understanding other amyloid proteins which are believed to be pathogenic as well in other amyloid-like protein such as spider silk and silk-worm silk. For instant, one can prevent the mechanically induced tau protein aggregation into amyloid in Alzheimer's disease patients by designing the smart drug that prevents the elongation of tau protein. The other smart design includes the drug that stabilizes the elongated state (beta sheet nuclei) and prevents further assembly of nanofiber core. While our experiment is done in vitro, molecular chaperone enzyme catalysts folding of protein in vivo by stretching it [81]. The other implication is that one can design the smart biomaterials in which shows superior toughness in certain direction by controlling the flow during selfassembly based on the fact that beta sheet is very tough in the parallel direction (tear mode) but modulate in perpendicular direction (shear mode) [23]. This concept is well illustrated by spider during silk spinning process. When concentrated protein solution is extruded through small spinneret, excessive shear force induced self-assembly of the beta sheet in the direction perpendicular to the shear flow direction. Later on the entire solution is dried out and results in spider fiber with extra toughness [82].

Considering the other application of this process, one can use this concept to create the nanoscale devices based on biomaterials such as protein fiber. Jonathan et. al., [29] enlightened the possibility of using nanomechanical stimuli to create the nanopattern made of nanofibril biomolecule with controllable directionality. However, in practical, this concept is complicated due to its stoichiometric nature of this process. The nanofibers were seemed to 
form randomly on the surface. Later on, Johnson et.al., [83] used different controlled AFM parameters, such as scan speed and scan line density, to gain better control on the nanofiber patterning and the nanofiber array pattern is successfully formed. Still, pattern of single nanofiber is challenging.

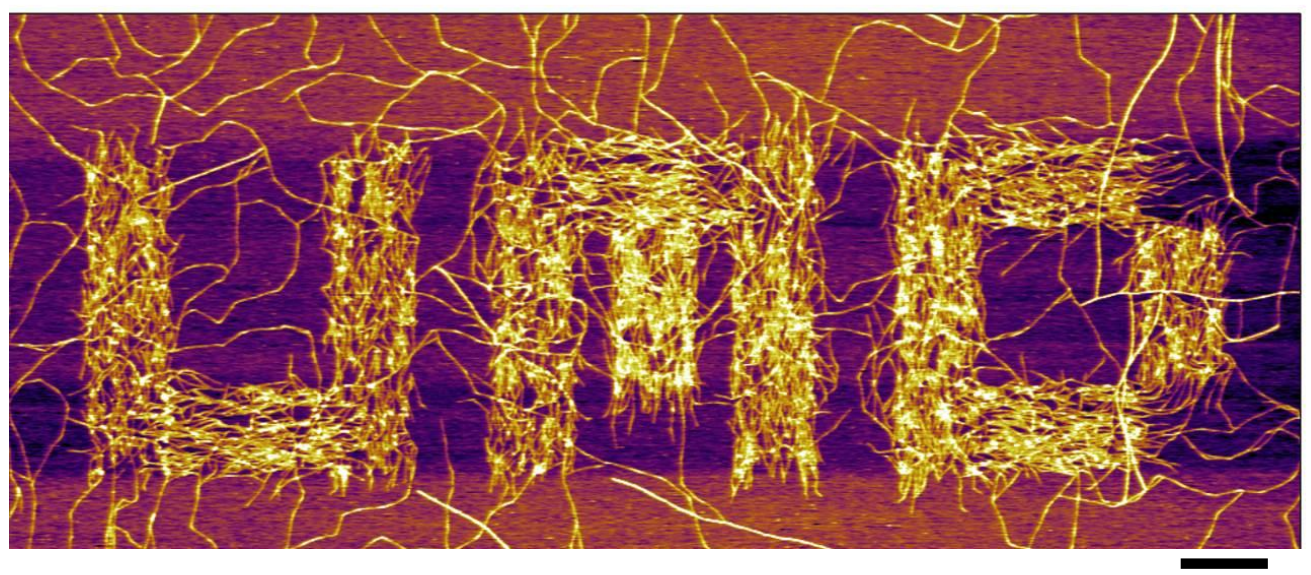

$1 \mu \mathrm{m}$

Figure 6-1. Patterning of SELP nanofiber arrays showed the potential as neuronal guidance circuit. In this case, the "UMD" pattern was created with the width of $1 \mu \mathrm{m}$ [83].

After elucidating the molecular mechanism of each individual nanofiber patterning as explained in the previous chapters, the individual single nanofiber can be created at specific location and controllable directionality.

\subsection{Experimental Methods}

Sample preparation follows the method explained previously. Lateral force microscopy (LFM) was performed. In order to create the single nanofiber, the tip is scanned along the single trajectory until the heightening area was found, indicate that the nucleation site was formed, and then stopped the single line scan. After one minute, the area scan was done on the single line scan to observe single nanofiber elongated perpendicular to the scan direction. 


\subsection{Single nanofiber patterning}

Using the method explained above, the single nanofiber patterning is successfully formed. The major complication is that the nucleation site usually resulted in two or more nanofibers which are not desirable. Close observation showed that the WLC curves tend to result in single nanofiber while sawtooth and exponential curves tend to yield multiple nanofibers. For future suggestion, one get better control on single nanofiber nucleation by slowing down the aggregation rate, for example, using low temperature sample. Utilizing tapping mode also minimizes the interaction between tip and the protein film and lesser chance of picking up multiple molecules.

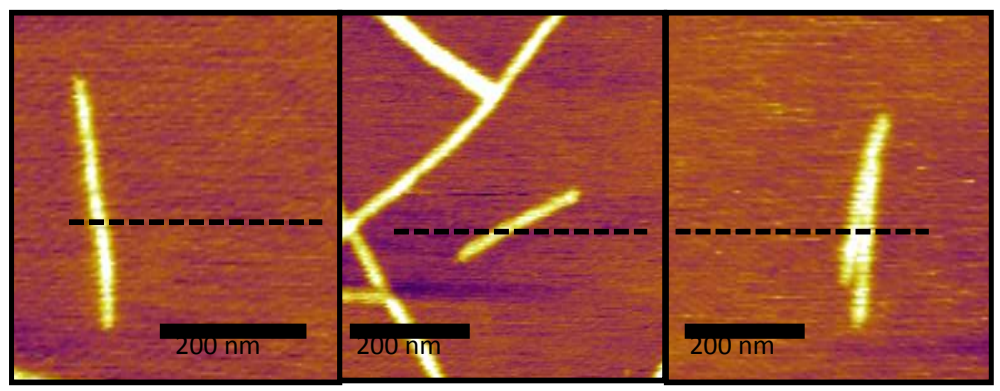

Figure 6-2. Single nanofiber pattern was created by repeatedly scan on the single line (as shown in dotted line) until the nucleation site was observed. After that, the area scan was performed on the line scan to reveal the single nanofiber elongated from the scan direction.

\section{$\underline{6.4 \text { Conclusion }}$}

In conclusion, we found that nanomechanical stretching of SELP lowers the activation energy of nucleation by increasing the lifetime of the extended conformation which acts as a template for nucleation of SELP amyloid on the mica substrate. Our direct observation of nucleus evolution revealed the stepwise increase in heights which matches the size of a beta hairpin structure in amyloid fibrils very well. The fact that the amyloid grows perpendicular to the stretching direction implies a guiding effect of the stretched state on the 
amyloid growth. Using this technique, a single nanofiber pattern was successfully created. The insights learned from this work may contribute to the design of a novel peptide system that can create a well-defined nanofiber patterned substrate and serve as a useful platform in the manufacturing of nanoscale functional devices. 


\section{Bibliography}

1. Merlini, G. and V. Bellotti, Molecular mechanisms of amyloidosis. N Engl J Med, 2003. 349(6): p. 583-96.

2. Dobson, C.M., Protein folding and misfolding. Nature, 2003. 426(6968): p. 884-890.

3. Fandrich, M. and C.M. Dobson, The behaviour of polyamino acids reveals an inverse side chain effect in amyloid structure formation. EMBO J, 2002. 21(21): p. 5682-90.

4. Fowler, D.M., et al., Functional amyloid-from bacteria to humans. Trends in biochemical sciences, 2007. 32(5): p. 217-224.

5. Knowles, T.P. and M.J. Buehler, Nanomechanics of functional and pathological amyloid materials. Nature nanotechnology, 2011. 6(8): p. 469479.

6. Scheibel, T., et al., Conducting nanowires built by controlled self-assembly of amyloid fibers and selective metal deposition. Proceedings of the National Academy of Sciences, 2003. 100(8): p. 4527-4532.

7. Thirumalai, D. and D.K. Klimov, Intermediates and transition states in protein folding. Methods Mol Biol, 2007. 350: p. 277-303.

8. Chan, H.S. and K.A. Dill, Protein folding in the landscape perspective: chevron plots and non-Arrhenius kinetics. Proteins, 1998. 30(1): p. 2-33.

9. Petkova, A.T., et al., Self-propagating, molecular-level polymorphism in Alzheimer's beta-amyloid fibrils. Science, 2005. 307(5707): p. 262-5.

10. Jarrett, J.T., E.P. Berger, and P.T. Lansbury, Jr., The carboxy terminus of the beta amyloid protein is critical for the seeding of amyloid formation: implications for the pathogenesis of Alzheimer's disease. Biochemistry, 1993. 32(18): p. 4693-7.

11. Nielsen, L., et al., Effect of environmental factors on the kinetics of insulin fibril formation: elucidation of the molecular mechanism. Biochemistry, 2001. 40(20): p. 6036-46.

12. Abrahamson, M. and A. Grubb, Increased body temperature accelerates aggregation of the Leu-68-->Gln mutant cystatin $C$, the amyloid-forming protein in hereditary cystatin $C$ amyloid angiopathy. Proc Natl Acad Sci U S A, 1994. 91(4): p. 1416-20.

13. Kowalewski, T. and D.M. Holtzman, In situ atomic force microscopy study of Alzheimer's beta-amyloid peptide on different substrates: New insights into mechanism of beta-sheet formation. Proceedings of the National Academy of Sciences of the United States of America, 1999. 96(7): p. 3688-3693.

14. Zhu, M., et al., Surface-catalyzed amyloid fibril formation. Journal of Biological Chemistry, 2002. 277(52): p. 50914-50922.

15. Hoyer, W., et al., Rapid self-assembly of alpha-synuclein observed by in situ atomic force microscopy. J Mol Biol, 2004. 340(1): p. 127-39.

16. McMasters, M.J., R.P. Hammer, and R.L. McCarley, Surface-induced aggregation of beta amyloid peptide by omega-substituted alkanethiol monolayers supported on gold. Langmuir, 2005. 21(10): p. 4464-4470. 
17. Ha, C. and C.B. Park, Ex situ atomic force microscopy analysis of betaamyloid self-assembly and deposition on a synthetic template. Langmuir, 2006. 22(16): p. 6977-6985.

18. Nayak, A., A.K. Dutta, and G. Belfort, Surface-enhanced nucleation of insulin amyloid fibrillation. Biochemical and Biophysical Research Communications, 2008. 369(2): p. 303-307.

19. Arimon, M., et al., Fine structure study of Abetal-42 fibrillogenesis with atomic force microscopy. FASEB J, 2005. 19(10): p. 1344-6.

20. Yokoi, H., T. Kinoshita, and S. Zhang, Dynamic reassembly of peptide RADA16 nanofiber scaffold. Proc Natl Acad Sci U S A, 2005. 102(24): p. 8414-9.

21. So, M., et al., Ultrasonication-dependent acceleration of amyloid fibril formation. J Mol Biol, 2011. 412(4): p. 568-77.

22. Stathopulos, P.B., et al., Sonication of proteins causes formation of aggregates that resemble amyloid. Protein Sci, 2004. 13(11): p. 3017-27.

23. Keten, S. and M.J. Buehler, Geometric confinement governs the rupture strength of H-bond assemblies at a critical length scale. Nano Letters, 2008. 8(2): p. 743-748.

24. Lee, J.S., J. Ryu, and C.B. Park, High-Throughput Analysis of Alzheimer's $\beta$ Amyloid Aggregation Using a Microfluidic Self-Assembly of Monomersf. Analytical Chemistry, 2009. 81(7): p. 2751-2759.

25. Hill, E.K., et al., Shear flow induces amyloid fibril formation. Biomacromolecules, 2006. 7(1): p. 10-13.

26. Vollrath, F. and D.P. Knight, Liquid crystalline spinning of spider silk. Nature, 2001. 410(6828): p. 541-8.

27. Rammensee, S., et al., Assembly mechanism of recombinant spider silk proteins. Proceedings of the National Academy of Sciences, 2008. 105(18): p. 6590-6595.

28. Xue, W.F., S.W. Homans, and S.E. Radford, Systematic analysis of nucleation-dependent polymerization reveals new insights into the mechanism of amyloid self-assembly. Proc Natl Acad Sci U S A, 2008. 105(26): p. 892631.

29. Chang, J., et al., Nanomechanical Stimulus Accelerates and Directs the SelfAssembly of Silk-Elastin-like Nanofibers. Journal of the American Chemical Society, 2011. 133(6): p. 1745-1747.

30. Siediecki, C., et al., Shear-dependent changes in the three-dimensional structure of human von Willebrand factor. Blood, 1996. 88(8): p. 2939-2950.

31. Forsey, R.W., et al., The effect of hyaluronic acid and phospholipid based lubricants on friction within a human cartilage damage model. Biomaterials, 2006. 27(26): p. 4581-4590.

32. Sills, S., et al., Molecular Origins of Elastomeric Friction

Fundamentals of Friction and Wear, E. Gnecco and E. Meyer, Editors. 2007, Springer Berlin Heidelberg. p. 659-676.

33. Liu, Y.H., T. Wu, and D.F. Evans, Lateral Force Microscopy Study on the Shear Properties of Self-Assembled Monolayers of Dialkylammonium Surfactant on Mica. Langmuir, 1994. 10(7): p. 2241-2245. 
34. Noy, A., et al., Chemical Force Microscopy - Exploiting Chemically-Modified Tips to Quantify Adhesion, Friction, and Functional-Group Distributions in Molecular Assemblies. Journal of the American Chemical Society, 1995. 117(30): p. 7943-7951.

35. Bhushan, B. and J. Utter, Nanoscale adhesion, friction and wear of proteins on polystyrene. Colloids and Surfaces B: Biointerfaces, (0).

36. Haugstad, G., et al., Probing Molecular Relaxation on Polymer Surfaces with Friction Force Microscopy. Langmuir, 1995. 11(9): p. 3473-3482.

37. Chernyak, Y.B. and A.I. Leonov, On the theory of the adhesive friction of elastomers. Wear, 1986. 108(2): p. 105-138.

38. Dandu, R., et al., Silk-elastinlike protein polymer hydrogels: Influence of monomer sequence on physicochemical properties. Polymer, 2009. 50(2): p. 366-374.

39. Ner, Y., et al., Electrospinning nanoribbons of a bioengineered silk-elastinlike protein (SELP) from water. Polymer, 2009. 50(24): p. 5828-5836.

40. Hwang, W., et al., Surface Induced Nanofiber Growth by Self-Assembly of a Silk-Elastin-like Protein Polymer. Langmuir, 2009. 25(21): p. 12682-12686.

41. Chang, J., et al., Nanomechanical Stimulus Accelerates and Directs the SelfAssembly of Silk-Elastin-like Nanofibers. J Am Chem Soc, 2011.

42. Johnson, S., et al., Directed patterning of the self-assembled silk-elastin-like nanofibers using a nanomechanical stimulus. Chem Commun (Camb), 2012. 48(86): p. 10654-6.

43. Dahlgren, P.R., et al., Atomic force microscopy analysis of the Huntington protein nanofibril formation. Nanomedicine, 2005. 1(1): p. 52-7.

44. McAllister, C., et al., Protein interactions and misfolding analyzed by AFM force spectroscopy. J Mol Biol, 2005. 354(5): p. 1028-42.

45. Goldsbury, C., et al., Watching amyloid fibrils grow by time-lapse atomic force microscopy. Journal of Molecular Biology, 1999. 285(1): p. 33-39.

46. Chen, C.L., et al., In situ AFM study of amelogenin assembly and disassembly dynamics on charged surfaces provides insights on matrix protein selfassembly. J Am Chem Soc, 2011. 133(43): p. 17406-13.

47. Alsteens, D., et al., Unzipping a functional microbial amyloid. ACS Nano, 2012. 6(9): p. 7703-11.

48. Follmer, C., et al., Dopamine affects the stability, hydration, and packing of protofibrils and fibrils of the wild type and variants of alpha-synuclein. Biochemistry, 2007. 46(2): p. 472-82.

49. Li, S.C., et al., Alpha-helical, but not beta-sheet, propensity of proline is determined by peptide environment. Proc Natl Acad Sci U S A, 1996. 93(13): p. 6676-81.

50. Thompson, J.B., et al., The backbone conformational entropy of protein folding: Experimental measures from atomic force microscopy. Journal of Molecular Biology, 2002. 322(3): p. 645-652.

51. Li, H.B., et al., Reverse engineering of the giant muscle protein titin. Nature, 2002. 418(6901): p. 998-1002.

52. Becker, N., et al., Molecular nanosprings in spider capture-silk threads. Nature Materials, 2003. 2(4): p. 278-283. 
53. Lee, G., et al., Nanospring behaviour of ankyrin repeats. Nature, 2006. 440(7081): p. 246-249.

54. Zhang, W.K., et al., Single-molecule force spectroscopy on Bombyx mori silk fibroin by atomic force microscopy. Langmuir, 2000. 16(9): p. 4305-4308.

55. Urry, D.W., et al., Elastin: a representative ideal protein elastomer. Philosophical Transactions of the Royal Society of London Series BBiological Sciences, 2002. 357(1418): p. 169-184.

56. Heinz, W.F. and J.H. Hoh, Spatially resolved force spectroscopy of biological surfaces using the atomic force microscope. Trends in biotechnology, 1999. 17(4): p. 143-150.

57. Seog, J., et al., Direct measurement of glycosaminoglycan intermolecular interactions via high-resolution force spectroscopy. Macromolecules, 2002. 35(14): p. 5601-5615.

58. Ogletree, D.F., R.W. Carpick, and M. Salmeron, Calibration of frictional forces in atomic force microscopy. Review of Scientific Instruments, 1996. 67(9): p. 3298-3306.

59. Varenberg, M., I. Etsion, and G. Halperin, An improved wedge calibration method for lateral force in atomic force microscopy. Review of Scientific Instruments, 2003. 74(7): p. 3362-3367.

60. Valiaev, A., et al., Hydration and conformational mechanics of single, endtethered elastin-like polypeptides. J Am Chem Soc, 2008. 130(33): p. 1093946.

61. Urry, D.W., et al., Elastin: a representative ideal protein elastomer. Philos Trans R Soc Lond B Biol Sci, 2002. 357(1418): p. 169-84.

62. Geisler, M., et al., Controlling the Structure of Proteins at Surfaces. J Am Chem Soc, 2010.

63. Ou-Yang, C. and S.H. Wang, Transfer an Epc Based Process Model into a Hierarchical Functional Model to Support Value Chain Development. International Journal of Innovative Computing Information and Control, 2012. 8(6): p. 4189-4203.

64. Rief, M., et al., Reversible unfolding of individual titin immunoglobulin domains by AFM. Science, 1997. 276(5315): p. 1109-12.

65. Keten, S. and M.J. Buehler, Nanostructure and molecular mechanics of spider dragline silk protein assemblies. Journal of the Royal Society Interface, 2010. 7(53): p. 1709-1721.

66. Frisbie, C.D., et al., Functional group imaging by chemical force microscopy. Science, 1994. 265(5181): p. 2071-4.

67. Overney, R.M., et al., Force Microscopy Study of Friction and Elastic Compliance of Phase-Separated Organic Thin-Films. Langmuir, 1994. 10(4): p. 1281-1286.

68. Maeda, N., et al., Adhesion and friction mechanisms of polymer-on-polymer surfaces. Science, 2002. 297(5580): p. 379-382.

69. Holmberg, K., et al., Friction and wear of coated surfaces - scales, modelling and simulation of tribomechanisms. Surface \& Coatings Technology, 2007. 202(4-7): p. 1034-1049. 
70. Bo, Z., et al., Peptide friction in water nanofilm on mica surface. Chinese Physics B, 2012. 21(2): p. 026801.

71. Li, H., et al., Reverse engineering of the giant muscle protein titin. Nature, 2002. 418(6901): p. 998-1002.

72. Fantner, G.E., et al., Sacrificial bonds and hidden length: unraveling molecular mesostructures in tough materials. Biophys J, 2006. 90(4): p. 14118.

73. McAllister, C., et al., Protein interactions and misfolding analyzed by AFM force spectroscopy. Journal of Molecular Biology, 2005. 354(5): p. 10281042.

74. Ohgo, K., et al., Investigation of structural transition of regenerated silk fibroin aqueous solution by Rheo-NMR spectroscopy. J Am Chem Soc, 2008. 130(12): p. 4182-6.

75. Gong, Z., et al., Two distinct beta-sheet fibrils from silk protein. Chem Commun (Camb), 2009(48): p. 7506-8.

76. Dong, J., et al., Insulin assembly damps conformational fluctuations: Raman analysis of amide I linewidths in native states and fibrils. Journal of Molecular Biology, 2003. 330(2): p. 431-442.

77. Sharma, S., et al., Monitoring Protein Conformation along the Pathway of Chaperonin-Assisted Folding. Cell, 2008. 133(1): p. 142-153.

78. Anson, M.L., Protein Denaturation and the Properties of Protein Groups. Advances in Protein Chemistry, 1945. 2: p. 361-386.

79. Munoz, V. and J.M. Sanchez-Ruiz, Exploring protein-folding ensembles: A variable-barrier model for the analysis of equilibrium unfolding experiments. Proceedings of the National Academy of Sciences of the United States of America, 2004. 101(51): p. 17646-17651.

80. Sachs, F. and H. Lecar, Stochastic-Models for Mechanical Transduction. Biophysical Journal, 1991. 59(5): p. 1143-1145.

81. Beissinger, M. and J. Buchner, How chaperones fold proteins. Biol Chem, 1998. 379(3): p. 245-59.

82. Rammensee, S., et al., Assembly mechanism of recombinant spider silk proteins. Proceedings of the National Academy of Sciences of the United States of America, 2008. 105(18): p. 6590-6595.

83. Johnson, S., et al., Directed patterning of the self-assembled silk-elastin-like nanofibers using a nanomechanical stimulus. Chemical Communications, 2012. 48(86): p. 10654-10656. 